\title{
Chemical Abundance Analysis of Three $\alpha$-poor, Metal-poor Stars in the Ultrafaint Dwarf Galaxy Horologium I*
}

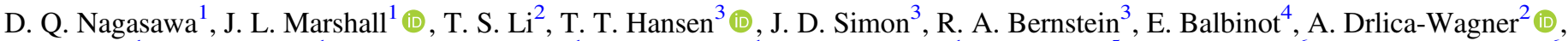
A. B. Pace ${ }^{1}$, L. E. Strigari ${ }^{1}$ (I) C. M. Pellegrino ${ }^{1}$, D. L. DePoy ${ }^{1}$, N. B. Suntzeff ${ }^{1}$, K. Bechtol ${ }^{5}$, A. R. Walker ${ }^{6}$,, T. M. C. Abbott ${ }^{6}$, F. B. Abdalla ${ }^{7,8}$, S. Allam ${ }^{2}$, J. Annis ${ }^{2}$ (1) , A. Benoit-Lévy ${ }^{7,9,10}$, E. Bertin ${ }^{9,10}$, D. Brooks ${ }^{7}$, A. Carnero Rosell ${ }^{11,12}$ (10), M. Carrasco Kind ${ }^{13,14}$, J. Carretero ${ }^{15}$, C. E. Cunha ${ }^{16}$, C. B. D'Andrea ${ }^{17}$, L. N. da Costa $^{11,12}$, C. Davis ${ }^{16}$, S. Desai ${ }^{18}$, P. Doel ${ }^{7}$, T. F. Eifler ${ }^{19,20}$, B. Flaugher ${ }^{2}$, P. Fosalba ${ }^{21}$, J. Frieman ${ }^{2,22}$, J. García-Bellido ${ }^{23}$, E. Gaztanaga ${ }^{21}$, D. W. Gerdes ${ }^{24,25}$ (1), D. Gruen ${ }^{16,26}$, , R. A. Gruendl ${ }^{13,14}$ (1) J. Gschwend ${ }^{11,12}$, G. Gutierrez ${ }^{2}$, W. G. Hartley ${ }^{7,27}$, K. Honscheid ${ }^{28,29}$, D. J. James ${ }^{30}$, T. Jeltema ${ }^{31}$, E. Krause ${ }^{16}$, K. Kuehn ${ }^{32}$, S. Kuhlmann ${ }^{33}$, N. Kuropatkin ${ }^{2}$, M. March ${ }^{17}$, R. Miquel ${ }^{15,34}$ (1) , B. Nord ${ }^{2}$, A. Roodman ${ }^{16,26}$, E. Sanchez ${ }^{35}$, B. Santiago ${ }^{11,36}$, V. Scarpine ${ }^{2}$, R. Schindler ${ }^{26}$, M. Schubnell ${ }^{25}$, I. Sevilla-Noarbe ${ }^{35}$, M. Smith $^{37}$, R. C. Smith ${ }^{6}$, M. Soares-Santos ${ }^{2}$ (1), F. Sobreira ${ }^{11,38}$, E. Suchyta ${ }^{39}$, G. Tarle ${ }^{25}$ (D), D. Thomas ${ }^{40}$, D. L. Tucker ${ }^{2}$,

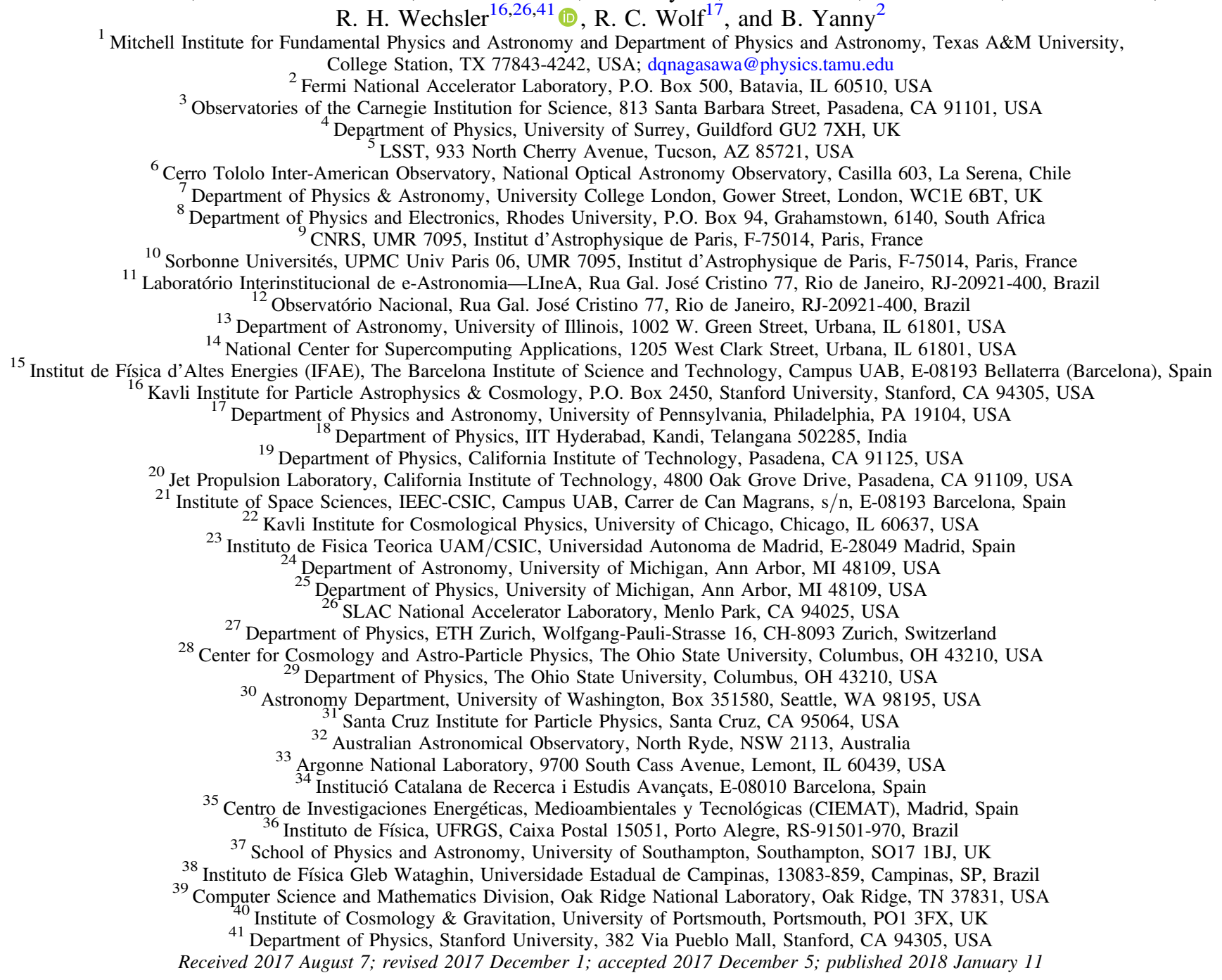
R. H. Wechsler ${ }^{16,26,41}$ (D) R. C. Wolf ${ }^{17}$, and B. Yanny ${ }^{2}$

\begin{abstract}
We present chemical abundance measurements of three stars in the ultrafaint dwarf galaxy Horologium I, a Milky Way satellite discovered by the Dark Energy Survey. Using high-resolution spectroscopic observations, we measure the metallicity of the three stars, as well as abundance ratios of several $\alpha$-elements, iron-peak elements, and neutron-capture elements. The abundance pattern is relatively consistent among all three stars, which have a
\end{abstract}

* This paper includes data gathered with the $6.5 \mathrm{~m}$ Magellan Telescopes located at Las Campanas Observatory, Chile. This paper also includes data based on observations made with the ESO Very Large Telescope at Paranal Observatory, Chile (ID 096.D-0967(B); PI: E. Balbinot). 
low average metallicity of $[\mathrm{Fe} / \mathrm{H}] \sim-2.6$ and are not $\alpha$-enhanced $([\alpha / \mathrm{Fe}] \sim 0.0)$. This result is unexpected when compared to other low-metallicity stars in the Galactic halo and other ultrafaint dwarfs and suggests the possibility of a different mechanism for the enrichment of Hor I compared to other satellites. We discuss possible scenarios that could lead to this observed nucleosynthetic signature, including extended star formation, enrichment by a Population III supernova, and or an association with the Large Magellanic Cloud.

Key words: galaxies: abundances - galaxies: dwarf - stars: abundances - stars: chemically peculiar

Supporting material: machine-readable table

\section{Introduction}

The past several decades have seen marked advancement in our understanding of how a galaxy like the Milky Way is assembled, as well as how chemical enrichment processes could have evolved to produce the elements that now exist in the local universe (e.g., Belokurov 2013; Frebel \& Norris 2015). From the early observational work of Searle \& Zinn (1978) a picture emerged that galaxies like the Milky Way most likely formed, at least in part, via hierarchical merging of smaller satellites. Modern dark energy+cold dark matter ( $\Lambda \mathrm{CDM}) \mathrm{N}$-body simulations of the Milky Way support this picture (e.g., Bullock \& Johnston 2005; Robertson et al. 2005; Johnston et al. 2008).

The past two decades have produced an abundance of new studies to compare to theory. Most of the progress in this field has been made through modern wide-field imaging surveys and subsequent spectroscopic study of the objects found in the survey images. For example, the Sloan Digital Sky Survey (SDSS) discovered many nearby "ultrafaint" dwarf galaxies in the Milky Way halo that have lower masses and higher massto-light ratios than previously known Milky Way satellites (see McConnachie 2012, for a summary). More recently, new widefield imaging surveys such as Pan-STARRS and the Dark Energy Survey (DES; The Dark Energy Survey Collaboration 2005) have discovered even more Milky Way satellite galaxies. DES has been the most prolific of these surveys to date: the first $2 \mathrm{yr}$ of DES data alone have resulted in the discovery of 22 new candidate satellites located in and around the Milky Way halo (Bechtol et al. 2015; Drlica-Wagner et al. 2015; Kim \& Jerjen 2015; Kim et al. 2015; Koposov et al. 2015; Luque et al. 2016a, 2016b). Once discovered, these candidates must be confirmed through kinematics to be gravitationally bound stellar associations via follow-up spectroscopic observations. Spectroscopic velocity measurements also yield a measure of the mass-to-light $(M / L)$ ratio and a determination of whether a satellite is a dark-matter-dominated dwarf galaxy or a baryondominated stellar cluster (see Willman \& Strader 2012, for a comprehensive definition). The DES-discovered candidate satellites considered most likely to be nearby ultrafaint dwarf galaxies have been selected for follow-up spectroscopy; six have subsequently been confirmed to be highly dark-matterdominated, low-luminosity satellites: Reticulum II (Ret II; Koposov et al. 2015; Simon et al. 2015a), Tucana II (Tuc II) and Grus I (Walker et al. 2016), Tucana III (Tuc III; Simon et al. 2017), Eridanus II (Li et al. 2017), and Horologium I (Hor I; Koposov et al. 2015), the last being the subject of this paper.

Due to their relative physical and therefore presumed chemical isolation at the time their stars were formed, ultrafaint dwarf galaxies provide opportunities to study not only the dark matter that dominates their mass profile but also the nucleosynthetic processes that occurred in the early universe. Star formation in these low-mass objects is likely to be highly influenced by only a few nucleosynthetic events (e.g., Ji et al. 2015). And since star formation in ultrafaint dwarfs appears to have been quenched early in the history of the universe, perhaps by reionization (Brown et al. 2014; Wetzel et al. 2015; Jeon et al. 2017), a fossil record of the early star formation history of these objects is preserved today.

Prior to the work presented here, three DES-discovered ultrafaint dwarfs have been the targets of detailed chemical study: Ret II, Tuc II, and Tuc III. In each of these galaxies a unique nucleosynthetic process is observed. The majority of stars in Ret II that have been studied to date are so-called $r$-II stars, signifying that they show extreme enhancement in rapid neutron-capture elements (Ji et al. 2016a; Roederer et al. 2016). This nucleosynthetic signature can be explained by a single high-yield event (e.g., a binary neutron star merger or hypernova) polluting the gas cloud from which stars in the galaxy were still forming. The chemical diversity of stars in Tuc II is somewhat unlike that observed in previously studied ultrafaint dwarfs and could be explained by a range of phenomena, not all of which follow the standard nucleosynthetic processes (Ji et al. 2016b). Hansen et al. (2017) report the discovery of a moderately $r$-process-enhanced $(r$-I) star in Tuc III, a rare chemical signature when compared to the bulk of field stars in the Milky Way halo, though not as rare as $r$-II stars. The diverse abundance patterns observed in these galaxies and the range of unusual phenomena invoked to explain them suggest that star formation in the early universe must have been a stochastic process that was highly variable on the mass scales of ultrafaint dwarf galaxies. If this trend holds for more of the newly discovered ultrafaint dwarfs, the study of chemical abundance patterns could provide an opportunity to improve our understanding of nucleosynthetic processes in the early universe.

In this paper we present a detailed chemical abundance analysis of the kinematically confirmed ultrafaint dwarf galaxy Hor I. Hor I is located at a heliocentric distance of $79 \mathrm{kpc}$, has a luminosity $M_{V} \sim-3.5 \pm 0.3 \mathrm{mag}$ (Bechtol et al. 2015), and has a mass-to-light ratio of $\sim 600$ (Koposov et al. 2015). The paper is organized as follows: In Section 2 we describe the observations and abundance analysis of three stars in Hor I. We present the abundance measurements in Section 3. In Section 4 we discuss the peculiar nature of the chemical abundance patterns observed in this galaxy. In Section 5 we conclude with a summary of the results and their impact.

\section{Observations and Data Analysis}

\subsection{Observations and Data Reduction}

Observations were performed using the FLAMES-UVES spectrograph (Dekker et al. 2000; Pasquini et al. 2000) on the VLT in Paranal, Chile, as part of program 096.D-0967(B) (PI: E. Balbinot) and the MIKE spectrograph (Bernstein et al. 2003) at the Magellan-Clay Telescope at Las Campanas Observatory 


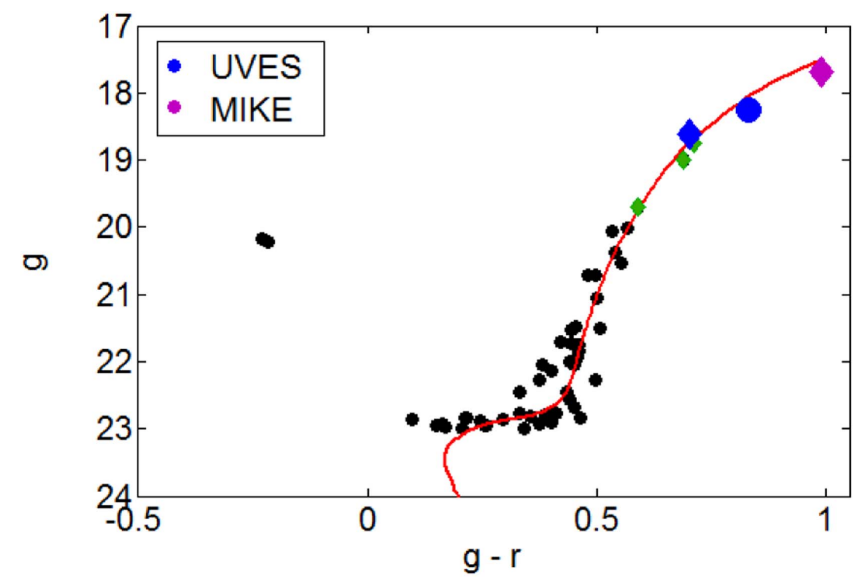

Figure 1. Color-magnitude diagram of high-probability $(>70 \%)$ candidate member stars of Hor I from Bechtol et al. (2015). A Dartmouth isochrone (Dotter et al. 2008) for a stellar population having $\tau=12.5 \mathrm{Gyr}$, $[\mathrm{Fe} / \mathrm{H}]=-2.5,[\alpha / \mathrm{Fe}]=+0.0$, and distance modulus $m-M=19.7$ as derived by Bechtol et al. (2015) is overplotted. The three stars studied in this work are indicated by larger points. The five diamond-shaped points are the confirmed member stars of Koposov et al. (2015). Black points are unconfirmed member stars from Bechtol et al. (2015).

(PI: R. Bernstein). In Figure 1 we present a color-magnitude diagram of the confirmed (Koposov et al. 2015) and highprobability (Bechtol et al. 2015) member stars of Hor I, constructed using photometry from DES. DES astrometry and photometry of the three stars studied in this paper are presented in Table 1.

\subsubsection{UVES Observations}

UVES observations took place on five nights over the months of 2015 December to 2016 January in 14 40-minute exposures. Stars were selected for UVES observation based on DES photometry, prior confirmation from Koposov et al. (2015), and considerations related to fiber positioning due to simultaneous observations with the FLAMES-GIRAFFE spectrograph (T. Li et al. 2017, in preparation). Two stars were selected for UVES observations: DES J025540-540807, a confirmed member from previous observations using mediumresolution spectra (Koposov et al. 2015), and DES J025543544349 , determined to be a likely member of Hor I (Bechtol et al. 2015). Spectra of UVES targets were obtained in service mode. The $580 \mathrm{~nm}$ configuration was used, resulting in wavelength coverage of $4800 \AA<\lambda<6800 \AA$ with a $\sim 30 \AA$ gap in coverage around $5800 \AA$ due to the CCD chip gap. We obtain a spectral resolution of $R \sim 47,000$.

Bias subtraction, flat-fielding, and spectral extraction were completed using the FLAMES-UVES Data Pipeline provided by the European Southern Observatory (Modigliani et al. 2004). Pixel oversampling (5 pixels per resolution element in the output spectrum) of the UVES spectra allowed us to boxcar-smooth the extracted spectra by 3 pixels in the wavelength dimension using the IRAF task boxcar without sacrificing information.

Radial velocities were measured via Fourier cross-correlation of each exposure using the IRAF task fxcor with a UVES spectrum of radial velocity standard HD 140283 observed on a different night (2012 May 29) with the same instrument settings as our observations. We take the statistical error to be the standard deviation of the resulting velocities derived for each of the 14 spectra, divided by the square root of the number of exposures (14). A correction was applied based on the date of the observation to shift the radial velocities to the heliocentric frame. Each exposure was then shifted to rest wavelength, and the 14 spectra were mean-combined using $3 \sigma$ rejection.

We estimate the systematic error of the radial velocities as follows. All spectra for a single star obtained on a given night were median-combined and then Fourier-cross-correlated with the combined spectra for the same star obtained on another night. To minimize the influence of noise, this crosscorrelation was performed over the limited wavelength range of $5100 \AA<\lambda<5300 \AA$ centered on the strong Mg triplet lines. For DES J025540-540807, this night-to-night crosscorrelation yielded an average relative velocity of $0.51 \mathrm{~km} \mathrm{~s}^{-1}$ with respect to each other. For DES J025543-544349, the average relative velocity was $0.43 \mathrm{~km} \mathrm{~s}^{-1}$.

The signal-to-noise ratio $(\mathrm{S} / \mathrm{N})$ per resolution element of the two UVES spectra and measured radial velocities are presented in Table 2. The reported radial velocity error is the quadrature combination of the statistical and systematic errors. We note that the velocity of DES J025543-544349 is consistent with the other stars in Hor I, increasing the number of confirmed Hor I member stars from five to six.

\subsubsection{MIKE Observations}

MIKE observations of DES J025535-540643, a confirmed Hor I member star (Koposov et al. 2015), took place on 2016 August 06 in five 30-minute exposures. Using a 0.7 arcsec slit and $2 \times 2$ pixel binning, the resulting spectrum has a resolution of $R \sim 22,000(\Delta \lambda=0.23 \AA)$ with coverage in the range of $3310 \AA<\lambda<5000 \AA$ for the blue chip and $4825 \AA<\lambda<9150 \AA$ for the red chip. Reduction of the data, including bias correction, flat-fielding, spectral extraction, wavelength calibration, and stacking, were completed using the MIKE pipeline (Kelson 2003).

For the spectrum obtained with MIKE, the radial velocity was measured by performing Fourier cross-correlation of the target star with a spectrum of radial velocity standard HD 146051 (radial velocity from Massarotti et al. 2008) observed on the same night using the IRAF task fxcor. A correction was applied based on the date of the observation to shift the radial velocities to the heliocentric frame. Each spectral order was considered individually; the reported radial velocity is the average value of the velocity measured in each order, and the reported error is the standard deviation of the radial velocities determined in each order of the spectrum. The measured S/N per resolution element and radial velocity for DES J025535540643 are presented in Table 2.

\subsection{Abundance Analysis}

We measured the equivalent widths of spectral features using the SPECTRE program ${ }^{42}$ (Sneden et al. 2012), with confirmation of the measurement of each line using the IRAF task splot. The line list was generated from the Kurucz database (Kurucz 2011) with updated laboratory transition probabilities from the NIST database (Kramida et al. 2016). Excitation potential, oscillator strength, and original laboratory source references for each line used in this analysis are listed in Table 3. For this analysis, it is assumed that these species are in local

\footnotetext{
${ }^{42}$ See http://www.as.utexas.edu/ chris/spectre.html for the most up-to-date version of SPECTRE.
} 
Table 1

DES Astrometry and Photometry of Three Member Stars of Hor I

\begin{tabular}{lccccrr}
\hline \hline ID & $\begin{array}{c}\text { R.A. (2000) } \\
(\mathrm{deg})\end{array}$ & $\begin{array}{c}\text { Decl. (2000) } \\
(\mathrm{deg})\end{array}$ & $g$ & $r$ & $i$ & \\
& 43.91793 & -54.13534 & 18.67 & 17.94 & 17.67 & 17.51 \\
DES J025540-540807 $^{\mathrm{a}}$ & 43.93246 & -54.08878 & 18.30 & 17.45 & 17.14 & 16.96 \\
DES J025543-544349 $_{\text {DES J025535-540643 }}^{\mathrm{b}}$ & 43.89665 & -54.11222 & $17.73^{\mathrm{c}}$ & 16.71 & 16.35 & 16.14 \\
\hline
\end{tabular}

Notes.

${ }^{a}$ Referred to as Horo 9 by Koposov et al. (2015).

${ }^{b}$ Referred to as Horo 10 by Koposov et al. (2015).

${ }^{c}$ Note that Koposov et al. (2015) report $g=19.31 \mathrm{mag}$ for this star.

Table 2

Observing Details

\begin{tabular}{lcccc}
\hline \hline ID & Instr. & $\begin{array}{c}\mathrm{S} / \mathrm{N} \\
\text { at } 5300 \AA\end{array}$ & $\begin{array}{c}\mathrm{S} / \mathrm{N} \\
\text { at } 6300 \AA\end{array}$ & $\begin{array}{c}V_{\text {helio }} \\
\left(\mathrm{km} \mathrm{s}^{-1}\right)\end{array}$ \\
\hline DES J025540-540807 & UVES & 30 & 40 & $118.6 \pm 0.6$ \\
DES J025543-544349 & UVES & 35 & 40 & $114.3 \pm 0.5$ \\
DES J025535-540643 & MIKE & 20 & 20 & $116.9 \pm 0.5$ \\
\hline
\end{tabular}

Table 3

Atomic Line Data

\begin{tabular}{lccrl}
\hline \hline Species & $\begin{array}{c}\lambda \\
(\AA)\end{array}$ & $\begin{array}{c}\text { E.P. } \\
(\mathrm{eV})\end{array}$ & $\begin{array}{c}\log (g f) \\
(\mathrm{dex})\end{array}$ & References \\
\hline Fe I & 4045.81 & 1.48 & 0.28 & Kurucz (2011) \\
Fe I & 4063.59 & 1.56 & 0.06 & Kramida et al. (2016) \\
Fe I & 4071.74 & 1.61 & -0.02 & Kurucz (2011) \\
Fe I & 4147.67 & 1.48 & -2.10 & Kurucz (2011) \\
Fe I & 4216.18 & 0.00 & -3.36 & Kurucz (2011) \\
Fe I & 4250.13 & 2.47 & -0.41 & Kramida et al. (2016) \\
Fe I & 4260.47 & 2.40 & 0.08 & Kramida et al. (2016) \\
Fe I & 4415.12 & 1.61 & -0.62 & Kurucz (2011) \\
Fe I & 4427.31 & 0.05 & -3.04 & Kurucz (2011) \\
$\ldots$ & $\ldots$ & $\ldots$ & $\ldots$ & \\
Tb II & 4002.57 & 0.64 & -0.49 & Lawler et al. (2001b, 2001d) \\
Tb II & 4005.47 & 0.13 & -0.02 & Lawler et al. (2001b) \\
Tb II & 4752.53 & 0.00 & -0.55 & Lawler et al. (2001b) \\
Dy II & 3944.68 & 0.00 & 0.11 & Wickliffe et al. (2000) \\
Dy II & 4103.31 & 0.10 & -0.38 & Wickliffe et al. (2000) \\
Dy II & 4449.70 & 0.00 & -1.03 & Wickliffe et al. (2000) \\
Er II & 3896.23 & 0.06 & -0.12 & Lawler et al. (2008) \\
Er II & 3938.63 & 0.00 & -0.52 & Kurucz (2011) \\
Th II & 4019.13 & 0.00 & -0.65 & Kurucz (2011) \\
\hline
\end{tabular}

(This table is available in its entirety in machine-readable form.)

thermodynamic equilibrium (LTE). For $\mathrm{CH}$ and $\mathrm{CN}$, we use dissociation energies of $3.47 \mathrm{eV}$ (Masseron et al. 2014) and $7.72 \mathrm{eV}$ (Sneden et al. 2014), respectively.

\subsubsection{Determination of Stellar Parameters}

Stellar parameters were derived spectroscopically from Fe I and Fe II lines using the abfind package of the 2017 version of the MOOG program ${ }^{43}$ (Sneden 1973) and the $\alpha$-enhanced 1D plane-parallel Castelli-Kurucz model atmospheres (Castelli \&

\footnotetext{
$\overline{43}$ See http://www.as.utexas.edu/ chris/moog.html for the most up-to-date version of MOOG.
}

Kurucz 2004). ${ }^{44}$ We note that, although the stars studied here do not in fact turn out to be $\alpha$-enhanced, we choose to use the Kurucz $\alpha$-enhanced models for consistency with our previous and future work. From comparison tests using DES J025540540807 , which has an $[\mathrm{Fe} / \mathrm{H}]=-2.43$, we further note that at the lowest metallicities the differences between the $\alpha$-enhanced and non- $\alpha$-enhanced Kurucz models are minimal, generally resulting in $\sim 0.05$ dex additional change in the abundances (which is much smaller than our total adopted uncertainties).

For spectra obtained using MIKE, the abundance of every spectral feature bluer than $4500 \AA$ was calculated while accounting for continuum scattering (Sobeck et al. 2011). For species in which the majority of or all lines measured were bluer than $4500 \AA$ and therefore greatly affected by continuum scattering, this changed abundances by $\sim 0.1$ dex. For species where most of the lines measured were redder than $4500 \AA$, the contribution from continuum scattering does not change the abundance significantly $(<0.05 \mathrm{dex})$.

Using these models, we calculate an abundance for each Fe I and Fe II line individually. We take the mean abundance of all measured lines for each species to be the measured abundance and use the standard deviation of these abundances as a statistical error. The effective temperature was determined by iterating atmospheric models until there was no observed trend in calculated Fe I abundance with excitation potential of the Fe I lines. Surface gravity was determined by iterating until there was $1 \sigma$ agreement between abundances calculated for $\mathrm{Fe}$ I and Fe II. In several instances, Fe II lines were measurable but weak, which may contribute to a systematic error regarding the determined surface gravities. Microturbulence in the stellar atmosphere was determined by iterating microturbulent velocity until there was no observed trend in the calculated abundances of $\mathrm{Fe}$ I with the reduced equivalent width of the $\mathrm{Fe}$ I lines. The same was done for Fe II as well; the derived microturbulence for $\mathrm{Fe}$ II was consistent with that derived for Fe I. Due to the known discrepancy between spectroscopically derived and photometrically derived effective temperature for metal-poor giant stars, a correction to the effective temperature was applied following Frebel et al. (2013). This empirical correction based on spectroscopically measured effective temperature increases the effective temperature by $\sim 100$ $-200 \mathrm{~K}$. Surface gravity, microturbulence, and abundances were then recalculated using this new effective temperature. This changed the surface gravity by $\sim+0.4$ dex and microturbulence by $\sim+0.1 \mathrm{~km} \mathrm{~s}^{-1}$. The resulting abundances using these new parameters differed by $\sim 0.2$ dex. We determine the uncertainty in our stellar parameters by varying the stellar

\footnotetext{
$\overline{44}$ See http://kurucz.harvard.edu/grids.html for the Castelli-Kurucz model atmospheres.
} 
Table 4

Measured Stellar Parameters

\begin{tabular}{|c|c|c|c|c|c|}
\hline ID & $\begin{array}{l}T_{\text {eff }} \\
(\mathrm{K})\end{array}$ & $\begin{array}{c}\log (g) \\
(\operatorname{dex})\end{array}$ & $\begin{array}{c}v_{\text {micro }} \\
\left(\mathrm{km} \mathrm{s}^{-1}\right)\end{array}$ & $\begin{array}{c}{[\mathrm{Fe} / \mathrm{H}]} \\
(\mathrm{dex})\end{array}$ & $\begin{array}{c}{[\mathrm{Ca} / \mathrm{Fe}]} \\
\quad(\mathrm{dex})\end{array}$ \\
\hline DES J025540-540807 & $5000 \pm 100$ & $2.0 \pm 0.2$ & $0.8 \pm 0.5$ & $-2.43 \pm 0.13$ & $-0.07 \pm 0.15$ \\
\hline DES J025543-544349 & $4800 \pm 100$ & $1.5 \pm 0.2$ & $1.8 \pm 0.5$ & $-2.60 \pm 0.16$ & $+0.00 \pm 0.13$ \\
\hline DES J025535-540643 & $4500 \pm 100$ & $1.4 \pm 0.2$ & $3.5 \pm 0.5$ & $-2.83 \pm 0.12$ & $-0.02 \pm 0.21$ \\
\hline
\end{tabular}

model and examining the resulting trends in excitation potential and reduced equivalent width. We calculate the final $[\mathrm{Fe} / \mathrm{H}]$ of our stars from Fe I owing to the greater number of lines measured. Measured stellar parameters are presented in Table 4.

\subsubsection{Element Abundance Measurement Using Equivalent Widths}

In both UVES and MIKE spectra, equivalent widths were measured for several species with strong, unblended absorption lines: Fe I, Fe II, $\mathrm{Na}$ I, Mg I, and Ca I. For Fe I in particular, lines ranging across wavelength, excitation potential (E.P.), and transition probability $\log (g f)$ were sampled in order to minimize systematic bias in abundance calculations. For Na I, we used corrections determined by Lind et al. (2011) for the $\mathrm{Na}$ $\lambda 5895.93$ doublet to compensate for non-LTE effects.

Due to the greater wavelength coverage of the MIKE spectrum, $60 \mathrm{Fe}$ I lines were measurable, compared to the only 12 useful Fe I lines in the UVES data. To ensure that the reduced number of lines in the UVES spectra would not systematically bias our measurements, the 12 Fe I lines used in the UVES analysis were measured in the MIKE spectrum and analyzed separately from the full 60-line analysis. The difference between the two analyses in both stellar parameter determination and abundance measurement was within the uncertainties. We conclude then that the reduced number of lines in the UVES spectral analysis does not systematically affect the results.

\subsubsection{Element Abundance Measurement Using Synthetic Spectra}

Spectral synthesis was done for elements that did not have a large number of measurable lines owing to low $\mathrm{S} / \mathrm{N}$ or blending and for elements where hyperfine structure and/or isotopic shifts needed to be considered. Using the stellar parameters derived, we have used spectral synthesis to measure the abundances of multiple elements in all three stars, specifically Si I, Sc II, Ti I, CrI, MnI, Ni I, BaII, and Eu II. The increased wavelength coverage in the MIKE spectrum enables measurement of additional species in DES J025535-540643. For these measurements, multiple spectral lines were identified based on both their excitation potential and transition probability to be relatively strong (i.e., low excitation energies, high transition probabilities). Synthetic spectra were generated using the synth package of the MOOG program (Sneden 1973) for a $40 \AA$ window centered on the line of interest. The abundances of $\mathrm{Fe}$ and $\mathrm{Ca}$ from equivalent width analysis were used as input in the synthesis. Spectra were generated varying the abundance of the elements of interest in $[\mathrm{X} / \mathrm{H}]$ steps of $0.10-0.125 \mathrm{dex}$. A Gaussian function was utilized in the smoothing of the synthetic spectra, which was roughly what was expected based on spectrograph resolution. If available in the $40 \mathrm{~A}$ window, an Fe I or $\mathrm{Ca}$ I line was used to ensure that the Gaussian-smoothed synthetic spectrum using the equivalent-width-derived stellar parameters was able to reproduce the observational data, generally reproducing observational data to $\sim 0.10$ dex. Best-fit spectra were selected by eye based on the $\chi^{2}$ minimization output in MOOG. Synthesis was also used to confirm the abundances derived using equivalent width analysis. Upper limits were derived by comparisons to synthetic spectra. Models of varying element abundances were generated until a model produced a clear detection that would have been distinguishable from noise but is undetected in the observed spectrum of the star. Sample synthetic spectra for elements measured using equivalent width analysis and spectral synthesis can be found in Figure 2, overlaid onto the observed spectra.

Abundances are calculated as $\log _{10}\left(\epsilon_{X}\right)$, which is defined in Equation (1) in terms of number density $N_{X}$. For reference, $\log _{10}\left(\epsilon_{H}\right)$, where $N_{H}$ is the number density of hydrogen, is defined as 12:

$$
\log _{10}\left(\epsilon_{X}\right)=\log _{10}\left(\frac{N_{X}}{N_{H}}\right)+12 .
$$

Conversion into the more familiar $[\mathrm{X} / \mathrm{H}]$ notation is performed using Equation (2) using measurements of $\log _{10}\left(\epsilon_{X, \odot}\right)$ by Asplund et al. (2009). Calculation of $[\mathrm{X} / \mathrm{Fe}]$ is shown in Equation (3).

$$
\begin{gathered}
{[\mathrm{X} / \mathrm{H}]_{\star}=\log _{10}\left(\epsilon_{X, \star}\right)-\log _{10}\left(\epsilon_{X, \odot}\right)} \\
{[\mathrm{X} / \mathrm{Fe}]_{\star}=[\mathrm{X} / \mathrm{H}]_{\star}-[\mathrm{Fe} / \mathrm{H}]_{\star} .}
\end{gathered}
$$

In order to reduce systematic errors, we used Fe I to calculate $[\mathrm{X} / \mathrm{Fe}]$ for neutral species and Fe II to calculate $[\mathrm{X} / \mathrm{Fe}]$ for ionized species. We present chemical abundance measurements in Table 5. We list each species measured, the number of lines measured for that species $(N), \log _{10}\left(\epsilon_{X}\right)$, metallicity, elemental abundance compared to iron, total error on the measurement (see discussion in Section 2.3), and the method used to measure each species. For lines for which we could only determine an upper limit, the total $1 \sigma$ uncertainty was added to the measured limit, i.e., we attempt to report a conservative estimate of the upper limit. For the UVES spectra we attempted to measure the abundances of several other elements, including $\mathrm{Al}, \mathrm{Co}, \mathrm{Cu}$, $\mathrm{Nd}, \mathrm{Sr}, \mathrm{Yb}$, and $\mathrm{Zn}$, but could not obtain an upper limit better than $[\mathrm{X} / \mathrm{Fe}]<+4$ dex for these elements owing to the lack of strong lines in the UVES wavelength range.

\subsection{Error Analysis}

In order to determine the uncertainty in the abundance measurements, we employ a method similar to that in McWilliam \& Rich (1994) and account for the statistical and systematic errors separately. For lines measured using equivalent widths, we have calculated the mean abundance for multiple lines across excitation potential and transition probability space. We assume that the standard deviation from this mean abundance represents our statistical error that arises 

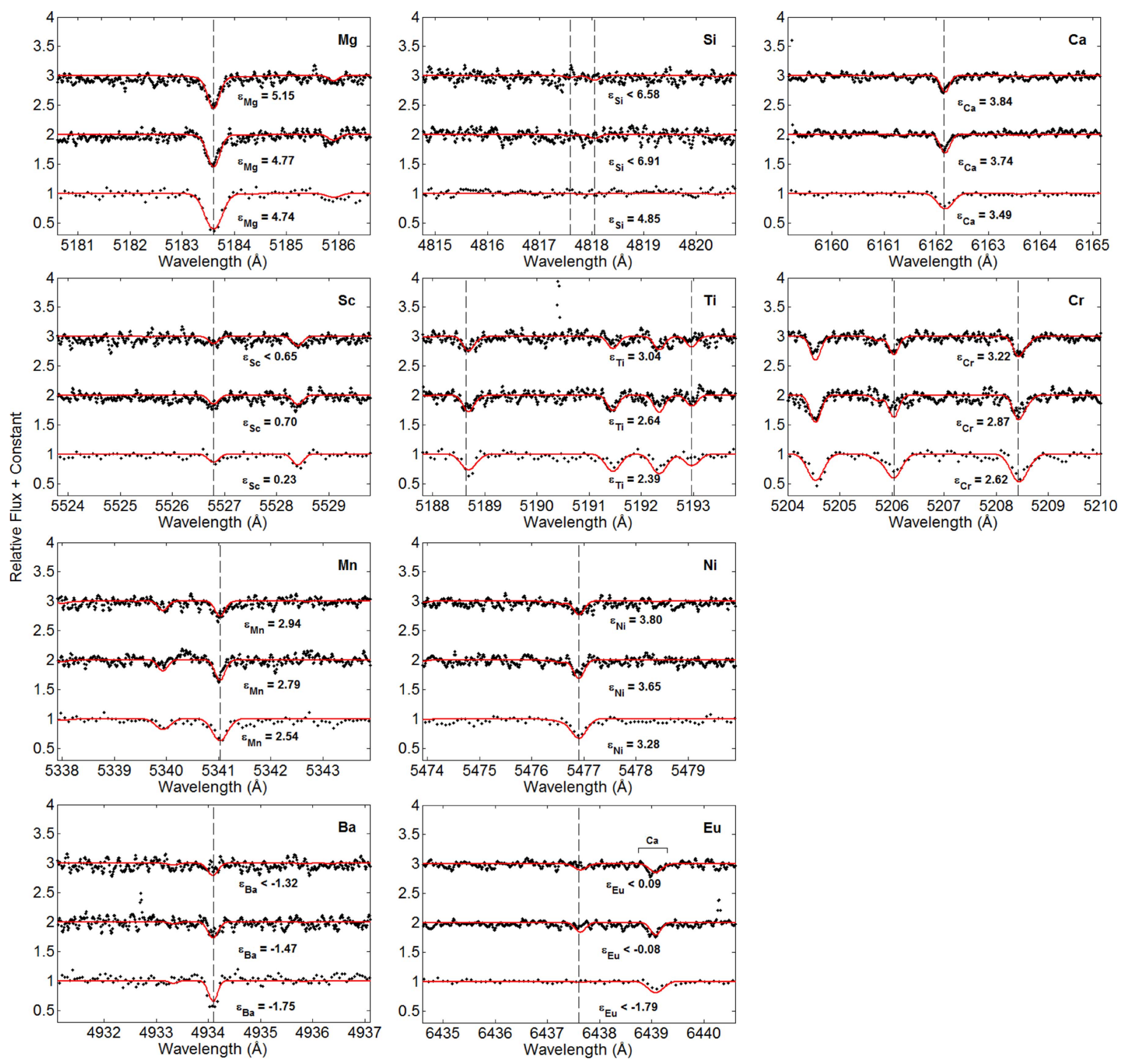

Figure 2. Examples of synthetic spectra showing the region around the absorption features for $\mathrm{Ca}, \mathrm{Mg}, \mathrm{Si}, \mathrm{Cr}, \mathrm{Mn}, \mathrm{Ti}, \mathrm{Sc}, \mathrm{Ni}, \mathrm{Ba}$, and Eu. In each panel, the top spectrum is DES J025540-540807, the middle spectrum is DES J025543-544349, and the bottom spectrum is DES J025535-540643. Observed data are plotted as black points, while synthetic spectra of the indicated $\epsilon_{X}$ are presented as red lines. Vertical dashed lines indicate the central wavelength of spectral features of the indicated element. It should be noted that the Si abundance for DES J025535-540643 was not derived from the doublet at 4817.58 and $4818.05 \AA$ alone; other lines outside of the wavelength coverage of UVES were used to achieve a positive detection. The wavelength coverage of UVES was used to achieve a positive detection.

from uncertainty in our equivalent width measurements. We take this to be the uncertainty on our abundance measurement for a single, unblended spectral feature. Therefore, by dividing by $\sqrt{N}$, where $N$ is the number of lines measured, we arrive at the statistical error in our abundance measurement that accounts for the multiple lines measured per element.

To account for systematic errors introduced by the uncertainty in stellar parameter determination, we vary the stellar atmosphere model by the uncertainty in the stellar parameters individually. We then recalculate the abundance of each element using this perturbed model and determine the variation in our abundance measurement $\Delta \log _{10}\left(\epsilon_{X}\right)$ caused by the perturbation. We do this for effective temperature $( \pm 100 \mathrm{~K})$, surface gravity $( \pm 0.2 \mathrm{dex})$, and microturbulence $\left( \pm 0.5 \mathrm{~km} \mathrm{~s}^{-1}\right)$.

The empirical effective temperature correction from Frebel et al. (2013) to account for the discrepancy between spectroscopically and photometrically derived temperatures may have increased the uncertainty in effective temperature. Since this correction was on order $\sim 100 \mathrm{~K}$, our measured error in effective temperature may be slightly larger, which may then affect the measured abundances. However, for the purposes of 
Table 5

Abundances of Three Confirmed Member Stars of Hor I

\begin{tabular}{|c|c|c|c|c|c|c|}
\hline Species & $N$ & $\log _{10}\left(\epsilon_{X}\right)$ & {$[\mathrm{X} / \mathrm{H}]$} & {$[\mathrm{X} / \mathrm{Fe}]$} & Error & Method \\
\hline \multicolumn{7}{|c|}{ DES J025540-540807 } \\
\hline $\mathrm{Na} I$ & 2 & 3.54 & -2.70 & -0.27 & 0.27 & Eq. Width \\
\hline Mg I & 2 & 5.15 & -2.45 & -0.02 & 0.25 & Eq. Width \\
\hline Si I & 4 & $<6.58$ & $<-0.93$ & $<+1.95$ & 0.45 & Spec. Synthesis \\
\hline $\mathrm{Ca} \mathrm{I}$ & 4 & 3.84 & -2.50 & -0.07 & 0.15 & Eq. Width \\
\hline Sc II & 1 & $<0.65$ & $<-2.50$ & $<+0.51$ & 0.44 & Spec. Synthesis \\
\hline Ti I & 3 & 3.04 & -1.91 & +0.52 & 0.40 & Spec. Synthesis \\
\hline Cr I & 8 & 3.22 & -2.42 & +0.01 & 0.30 & Spec. Synthesis \\
\hline Mn I & 3 & 2.94 & -2.49 & -0.06 & 0.61 & Spec. Synthesis \\
\hline Fe I & 12 & 5.07 & -2.43 & +0.00 & 0.13 & Eq. Width \\
\hline Fe II & 4 & 4.93 & -2.57 & -0.14 & 0.11 & Eq. Width \\
\hline Ni I & 2 & 3.80 & -2.42 & +0.01 & 0.41 & Spec. Synthesis \\
\hline Ba II & 3 & $<-1.32$ & $<-3.50$ & $<-0.47$ & 0.46 & Spec. Synthesis \\
\hline Eu II & 2 & $<0.09$ & $<-0.43$ & $<+2.55$ & 0.41 & Spec. Synthesis \\
\hline \multicolumn{7}{|c|}{ DES J025543-544349 } \\
\hline $\mathrm{Na} I$ & 2 & 2.74 & -3.50 & -0.90 & 0.32 & Eq. Width \\
\hline $\mathrm{Mg} \mathrm{I}$ & 3 & 4.77 & -2.83 & -0.23 & 0.25 & Eq. Width \\
\hline Si I & 4 & $<6.91$ & $<-0.60$ & $<+2.45$ & 0.45 & Spec. Synthesis \\
\hline $\mathrm{Ca} \mathrm{I}$ & 3 & 3.74 & -2.60 & +0.00 & 0.13 & Eq. Width \\
\hline Sc II & 1 & 0.70 & -2.45 & +0.27 & 0.50 & Spec. Synthesis \\
\hline Ti I & 3 & 2.64 & -2.31 & +0.29 & 0.40 & Spec. Synthesis \\
\hline Cr I & 8 & 2.87 & -2.77 & -0.17 & 0.31 & Spec. Synthesis \\
\hline Mn I & 3 & 2.79 & -2.64 & -0.04 & 0.68 & Spec. Synthesis \\
\hline $\mathrm{Fe} I$ & 12 & 4.90 & -2.60 & +0.00 & 0.16 & Eq. Width \\
\hline Fe II & 4 & 4.78 & -2.72 & -0.12 & 0.11 & Eq. Width \\
\hline Ni I & 2 & 3.65 & -2.57 & +0.03 & 0.47 & Spec. Synthesis \\
\hline Ba II & 3 & -1.47 & -3.65 & -0.93 & 0.32 & Spec. Synthesis \\
\hline Eu II & 2 & $<-0.08$ & $<-0.60$ & $<+2.59$ & 0.47 & Spec. Synthesis \\
\hline \multicolumn{7}{|c|}{ DES J025535-540643 } \\
\hline $\mathrm{C}(\mathrm{CH})$ & 1 & $<5.05$ & $<-3.38$ & $<-0.19$ & 0.36 & Spec. Synthesis \\
\hline $\mathrm{N}(\mathrm{CN})$ & 1 & $<5.70$ & $<-2.13$ & $<+1.20$ & 0.50 & Spec. Synthesis \\
\hline $\mathrm{Na} I$ & 2 & 2.38 & -3.86 & -1.03 & 0.23 & Eq. Width \\
\hline Mg I & 4 & 4.74 & -2.86 & -0.03 & 0.30 & Eq. Width \\
\hline $\mathrm{Al} \mathrm{I}$ & 2 & 2.62 & -3.83 & -1.00 & 0.22 & Spec. Synthesis \\
\hline Si I & 1 & 4.80 & -2.71 & +0.12 & 0.48 & Spec. Synthesis \\
\hline $\mathrm{Ca} \mathrm{I}$ & 4 & 3.49 & -2.85 & -0.02 & 0.21 & Eq. Width \\
\hline Sc II & 3 & 0.21 & -2.94 & +0.00 & 0.15 & Spec. Synthesis \\
\hline Ti I & 3 & 2.39 & -2.56 & +0.27 & 0.18 & Spec. Synthesis \\
\hline $\mathrm{V}_{\mathrm{I}}$ & 1 & 1.80 & -2.13 & +0.70 & 0.30 & Spec. Synthesis \\
\hline Cr I & 5 & 2.62 & -3.02 & -0.19 & 0.38 & Spec. Synthesis \\
\hline Mn I & 1 & 2.54 & -2.89 & -0.06 & 0.36 & Spec. Synthesis \\
\hline $\mathrm{Fe} I$ & 60 & 4.67 & -2.83 & +0.00 & 0.12 & Eq. Width \\
\hline Fe II & 4 & 4.56 & -2.94 & -0.11 & 0.19 & Eq. Width \\
\hline Co I & 3 & 2.38 & -2.61 & +0.22 & 0.32 & Spec. Synthesis \\
\hline Ni I & 3 & 3.28 & -2.94 & -0.11 & 0.35 & Spec. Synthesis \\
\hline $\mathrm{Cu} \mathrm{I}$ & 3 & $<1.16$ & $<-3.03$ & $<+0.12$ & 0.32 & Spec. Synthesis \\
\hline $\mathrm{Zn} \mathrm{I}$ & 2 & $<2.30$ & $<-2.26$ & $<+0.87$ & 0.30 & Spec. Synthesis \\
\hline Ga I & 1 & $<0.68$ & $<-2.36$ & $<+0.90$ & 0.43 & Spec. Synthesis \\
\hline $\mathrm{Rb} \mathrm{I}$ & 2 & $<2.30$ & $<-0.22$ & $<+2.95$ & 0.34 & Spec. Synthesis \\
\hline Sr II & 2 & -0.97 & -3.84 & -0.90 & 0.33 & Spec. Synthesis \\
\hline Y II & 4 & $<-0.06$ & $<-2.27$ & $<+1.05$ & 0.38 & Spec. Synthesis \\
\hline Zr II & 4 & $<0.70$ & $<-1.88$ & $<+1.40$ & 0.34 & Spec. Synthesis \\
\hline Mo II & 1 & $<0.52$ & $<-1.36$ & $<+1.89$ & 0.31 & Spec. Synthesis \\
\hline Ba II & 3 & -1.75 & -3.93 & -0.99 & 0.33 & Spec. Synthesis \\
\hline La II & 5 & $<-0.18$ & $<-1.28$ & $<+1.98$ & 0.32 & Spec. Synthesis \\
\hline Ce II & 5 & $<-0.45$ & $<-2.03$ & $<+1.21$ & 0.30 & Spec. Synthesis \\
\hline Pr II & 4 & $<-1.04$ & $<-1.76$ & $<+1.49$ & 0.31 & Spec. Synthesis \\
\hline Nd II & 6 & $<-0.50$ & $<-1.92$ & $<+1.33$ & 0.31 & Spec. Synthesis \\
\hline Sm II & 5 & $<-0.55$ & $<-1.51$ & $<+1.75$ & 0.32 & Spec. Synthesis \\
\hline Eu II & 4 & $<-1.84$ & $<-2.36$ & $<+0.97$ & 0.39 & Spec. Synthesis \\
\hline
\end{tabular}


Table 5

(Continued)

\begin{tabular}{|c|c|c|c|c|c|c|}
\hline Species & $N$ & $\log _{10}\left(\epsilon_{X}\right)$ & {$[\mathrm{X} / \mathrm{H}]$} & {$[\mathrm{X} / \mathrm{Fe}]$} & Error & Method \\
\hline Tb II & 3 & $<-0.47$ & $<-0.77$ & $<+2.52$ & 0.35 & Spec. Synthesis \\
\hline Dy II & 3 & $<-0.75$ & $<-1.85$ & $<+1.43$ & 0.34 & Spec. Synthesis \\
\hline
\end{tabular}

this paper, we adopt our stated errors solely based on the observed trends in abundance calculation across excitation potential and transition probability space.

The variation in abundance due to the perturbed stellar parameters is added in quadrature with the statistical error taken from the uncertainty in our equivalent width measurements, generating $\Delta \log _{10}\left(\epsilon_{X}\right)_{\text {, Total }}$.

For lines measured using spectral synthesis, we assess systematic errors as described above. However, because we use the consistency of multiple lines to measure element abundance, we cannot derive a statistical uncertainty in the same manner as the equivalent width analysis. We still remeasure abundances using a stellar atmosphere model perturbed by the uncertainty in the measured stellar parameters. Our perturbed model abundance is compared against the unperturbed abundance to determine the variation $\Delta \epsilon$, which we take to be our systematic errors based on the errors in our stellar parameter determination. We estimate, based on $\mathrm{S} / \mathrm{N}$ and the variations observed in our stellar parameter perturbation, that the statistical error associated with this measurement could be as high as 0.25 dex. We therefore adopt this value as the statistical error for lines measured using synthetic spectra. The final statistical error reported for lines measured using spectral synthesis is this value divided by the square root of the number of lines measured.

We present the results of our error analysis in Table 6. The final errors reported in column (6) of Table 5 and column (7) of Table 6 are the quadrature sum of the systematic and statistical errors.

\section{Results}

In Figure 3 we compare Hor I stars to stars in the Milky Way halo and 13 ultrafaint dwarf galaxies for which spectroscopic abundance analysis has been performed. The three Hor I stars are all of very low metallicity, in the range of $-2.83<[\mathrm{Fe} / \mathrm{H}]<-2.43$, and have similar $\alpha$-element and iron-peak element abundances. The measurement of Ba II in two stars and a consistent upper limit in the third star suggest that the abundance of neutron-capture elements in these three stars is also similar.

In comparison to most other stars in the Milky Way halo and in other ultrafaint dwarf galaxies, the $\alpha$-element abundance of these three Hor I stars is low for their $[\mathrm{Fe} / \mathrm{H}]$. This can be seen in the $[\mathrm{Ca} / \mathrm{Fe}]$ and $[\mathrm{Mg} / \mathrm{Fe}]$ abundances. The detection of $\mathrm{Si}$ in one star, DES J025535-540643, is also consistent with the other $\alpha$-elements. There are a few stars in other ultrafaint dwarfs with similarly low $[\mathrm{Ca} / \mathrm{Fe}]$ and $[\mathrm{Mg} / \mathrm{Fe}](\sim 0)$; however, these stars are generally more metal-rich, and no other ultrafaint dwarf has consistently low abundances for all $\alpha$-elements among all its measured member stars.

The iron-peak elements also present some unusual patterns. The abundances of $\mathrm{Sc}$ and $\mathrm{Ni}$ seem to be similar to those of stars in the halo and the other ultrafaint dwarfs. The abundance of $\mathrm{Cr}$ in Hor I is slightly elevated with respect to most other ultrafaint dwarfs, but still consistent with the abundances of halo stars. However, the abundance of $\mathrm{Mn}$ is $\sim 0.4$ dex higher than most halo stars and $\sim 0.6$ dex higher than the abundances found in other ultrafaint dwarfs.

The abundance of $\mathrm{Ba}$ is similar to most other ultrafaint dwarfs. It does not present significant $s$-process or $r$-process enrichment like the stars in Ret II (Ji et al. 2016c) or Tuc III (Hansen et al. 2017). The upper limit of Eu found in DES J025535-540643 ([Eu/Fe] $<+1.02)$ excludes it from being an $r$-II star (defined as $[\mathrm{Eu} / \mathrm{Fe}]>+1.0$ ) but does not exclude the possibility that it is an $r$-I star (defined as $[\mathrm{Eu} / \mathrm{Fe}]>+0.3$ ), where these definitions are taken from Beers \& Christlieb (2005). However, the low $[\mathrm{Ba} / \mathrm{Fe}]$ values of these three stars make it unlikely that they are $r$-process enhanced.

Due to wavelength constraints, we could only measure $\mathrm{C}$ using the $\mathrm{CH}$ band in DES J025535-540643. Based on the upper limit of $[\mathrm{C} / \mathrm{Fe}]<-0.14$, we can conclude that this star is not carbon-enhanced.

\section{Discussion}

We discuss possible scenarios that could lead to the observed nucleosynthetic pattern of Hor I and compare Hor I stars with stars in the Milky Way with similar abundance patterns. In Section 4.1, we compare the abundance pattern of Hor I with that of stars found in the Milky Way halo with similar nucleosynthetic patterns. In Section 4.2, we discuss one plausible enrichment scenario, the early onset of Type Ia supernovae (SNe Ia) in Hor I. In Section 4.3, we compare the peculiar abundance pattern observed in Hor I with theoretical nucleosynthetic yield models. In Section 4.4, we discuss a possible association with the Large Magellanic Cloud (LMC) as the cause of the abundance pattern measured in Hor I. We caution that these discussions are based on the abundance measurements of only three stars and may change with analysis of additional stars in Hor I.

\subsection{Comparison with Similarly Peculiar Stars in the Milky Way Halo}

The stars in Hor I are not the first metal-poor, $\alpha$-poor stars to be discovered. For example, in a detailed chemical abundance study of stars found in a search for the most metal-poor stars in the Galactic halo, Ivans et al. (2003) reported chemical abundance measurements of two additional low- $\alpha$, low-metallicity stars in the Galactic halo, G4-36 and CS 22966-043, and found that these two and BD $+80^{\circ} 245$ all have $[\mathrm{Fe} / \mathrm{H}] \sim-2$ and $[\mathrm{Ca} / \mathrm{Fe}] \sim 0.5$ dex below the mean halo value (Ivans et al. [2003] report $[\mathrm{Ca} / \mathrm{Fe}]=+0.31$ for the halo). Interestingly, these three stars also have iron-peak overabundances that are qualitatively similar to the Hor I stars, with $\mathrm{BD}+80^{\circ} 245$ having the most similar abundances to our stars. Additionally, BD $+80^{\circ} 245$ has a similarly high $\mathrm{Ti}$ abundance despite its low $\alpha$-element abundance. An emerging 
Table 6

Summary of Error Analysis

\begin{tabular}{|c|c|c|c|c|c|c|}
\hline Species & $N$ & $\sigma$ & $\begin{array}{c}\Delta \log _{10}\left(\epsilon_{X}\right) \\
(\Delta T=+100 \mathrm{~K})\end{array}$ & $\begin{array}{c}\Delta \log _{10}\left(\epsilon_{X}\right) \\
(\Delta \log (g)=+0.2 \text { dex })\end{array}$ & $\begin{array}{c}\Delta \log _{10}\left(\epsilon_{X}\right) \\
\left(\Delta \xi=+0.5 \mathrm{~km} \mathrm{~s}^{-1}\right)\end{array}$ & $\Delta \log _{10}\left(\epsilon_{X}\right)_{\text {,Total }}$ \\
\hline \multicolumn{7}{|c|}{ DES J025540-540807 } \\
\hline $\mathrm{Na} \mathrm{I}$ & 1 & 0.10 & +0.13 & -0.06 & -0.20 & 0.27 \\
\hline Mg I & 2 & 0.13 & +0.14 & -0.08 & -0.16 & 0.25 \\
\hline Si I & 4 & 0.25 & +0.25 & +0.25 & +0.25 & 0.45 \\
\hline $\mathrm{Ca} \mathrm{I}$ & 4 & 0.03 & +0.08 & -0.03 & -0.12 & 0.15 \\
\hline Sc II & 1 & 0.25 & +0.25 & +0.25 & -0.10 & 0.44 \\
\hline Ti I & 3 & 0.25 & -0.25 & +0.13 & -0.25 & 0.40 \\
\hline Cr I & 8 & 0.20 & -0.10 & -0.10 & -0.25 & 0.30 \\
\hline Mn I & 3 & 0.50 & +0.25 & +0.25 & +0.50 & 0.61 \\
\hline Fe I & 12 & 0.20 & +0.11 & -0.01 & -0.05 & 0.13 \\
\hline $\mathrm{Fe}$ II & 4 & 0.13 & +0.05 & +0.07 & -0.01 & 0.11 \\
\hline Ni I & 2 & 0.25 & +0.25 & +0.10 & -0.25 & 0.41 \\
\hline Ba II & 3 & 0.25 & -0.25 & -0.25 & -0.25 & 0.46 \\
\hline Eu II & 2 & 0.25 & -0.25 & -0.25 & -0.10 & 0.41 \\
\hline \multicolumn{7}{|c|}{ DES J025543-544349 } \\
\hline $\mathrm{Na} \mathrm{I}$ & 1 & 0.19 & +0.12 & -0.02 & -0.24 & 0.32 \\
\hline Mg I & 3 & 0.09 & +0.09 & -0.06 & -0.22 & 0.25 \\
\hline Si I & 4 & 0.25 & +0.25 & +0.25 & +0.25 & 0.45 \\
\hline $\mathrm{Ca} \mathrm{I}$ & 3 & 0.04 & +0.09 & -0.03 & -0.08 & 0.13 \\
\hline Sc II & 1 & 0.25 & +0.25 & +0.25 & +0.25 & 0.50 \\
\hline Ti I & 3 & 0.25 & -0.25 & -0.13 & -0.25 & 0.40 \\
\hline Cr I & 8 & 0.20 & -0.10 & -0.13 & -0.25 & 0.31 \\
\hline Mn I & 3 & 0.50 & +0.25 & +0.25 & +0.50 & 0.68 \\
\hline $\mathrm{Fe} I$ & 12 & 0.29 & +0.13 & -0.02 & -0.04 & 0.16 \\
\hline $\mathrm{Fe}$ II & 4 & 0.17 & -0.02 & +0.07 & -0.01 & 0.11 \\
\hline Ni I & 2 & 0.25 & +0.25 & -0.25 & -0.25 & 0.47 \\
\hline Ba II & 3 & 0.25 & -0.10 & -0.10 & -0.25 & 0.32 \\
\hline Eu II & 2 & 0.25 & -0.25 & -0.25 & -0.25 & 0.47 \\
\hline \multicolumn{7}{|c|}{ DES J025535-540643 } \\
\hline $\mathrm{C}(\mathrm{CH})$ & 1 & 0.25 & +0.15 & -0.05 & -0.20 & 0.36 \\
\hline $\mathrm{N}(\mathrm{CN})$ & 1 & 0.25 & +0.25 & +0.25 & +0.25 & 0.50 \\
\hline $\mathrm{Na} \mathrm{I}$ & 1 & 0.08 & +0.14 & -0.05 & -0.16 & 0.23 \\
\hline Mg I & 4 & 0.27 & +0.14 & -0.08 & -0.16 & 0.30 \\
\hline $\mathrm{Al} \mathrm{I}$ & 2 & 0.07 & +0.15 & -0.08 & +0.13 & 0.22 \\
\hline Si I & 1 & 0.25 & +0.30 & +0.20 & +0.20 & 0.48 \\
\hline $\mathrm{Ca} \mathrm{I}$ & 4 & 0.29 & +0.08 & -0.03 & -0.12 & 0.21 \\
\hline Sc II & 3 & 0.12 & +0.10 & +0.08 & -0.05 & 0.15 \\
\hline Ti I & 3 & 0.09 & +0.10 & -0.10 & -0.10 & 0.18 \\
\hline V I & 1 & 0.25 & +0.10 & +0.10 & +0.10 & 0.30 \\
\hline Cr I & 5 & 0.35 & -0.09 & -0.24 & -0.23 & 0.38 \\
\hline Mn I & 1 & 0.25 & +0.05 & -0.20 & -0.15 & 0.36 \\
\hline Fe I & 60 & 0.20 & +0.11 & -0.01 & -0.05 & 0.12 \\
\hline $\mathrm{Fe}$ II & 4 & 0.33 & +0.05 & +0.07 & -0.01 & 0.19 \\
\hline Co I & 3 & 0.29 & +0.22 & -0.13 & -0.08 & 0.32 \\
\hline Ni I & 3 & 0.47 & +0.17 & +0.12 & +0.08 & 0.35 \\
\hline $\mathrm{Cu} \mathrm{I}$ & 3 & 0.25 & +0.15 & +0.10 & -0.10 & 0.32 \\
\hline Zn I & 2 & 0.25 & +0.10 & +0.10 & +0.10 & 0.30 \\
\hline Ga I & 1 & 0.25 & +0.20 & +0.20 & +0.20 & 0.43 \\
\hline $\mathrm{Rb} \mathrm{I}$ & 2 & 0.25 & +0.20 & +0.10 & +0.05 & 0.34 \\
\hline Sr II & 2 & 0.25 & +0.13 & -0.08 & -0.15 & 0.33 \\
\hline Y II & 4 & 0.25 & +0.20 & +0.20 & +0.05 & 0.38 \\
\hline Zr II & 4 & 0.25 & -0.20 & +0.05 & +0.10 & 0.34 \\
\hline Mo II & 1 & 0.25 & +0.15 & -0.10 & +0.05 & 0.31 \\
\hline Ba II & 3 & 0.52 & +0.10 & +0.07 & -0.07 & 0.33 \\
\hline La II & 5 & 0.25 & +0.15 & +0.10 & +0.10 & 0.32 \\
\hline Ce II & 5 & 0.25 & +0.10 & -0.10 & -0.10 & 0.30 \\
\hline Pr II & 4 & 0.25 & +0.15 & +0.10 & +0.05 & 0.31 \\
\hline Nd II & 6 & 0.25 & +0.15 & -0.10 & -0.05 & 0.31 \\
\hline Sm II & 5 & 0.25 & +0.15 & -0.10 & -0.10 & 0.32 \\
\hline Eu II & 4 & 0.25 & -0.20 & -0.20 & -0.10 & 0.39 \\
\hline
\end{tabular}


Table 6

(Continued)

\begin{tabular}{lccccc}
\hline \hline Species & $N$ & $\sigma$ & $\begin{array}{c}\Delta \log _{10}\left(\epsilon_{X}\right) \\
(\Delta T=+100 \mathrm{~K})\end{array}$ & $\begin{array}{c}\Delta \log _{10}\left(\epsilon_{X}\right) \\
(\Delta \log (g)=+0.2 \text { dex })\end{array}$ & $\begin{array}{c}\Delta \log _{10}\left(\epsilon_{X}\right) \\
\left(\Delta \xi=+0.5 \mathrm{~km} \mathrm{~s}^{-1}\right)\end{array}$ \\
\hline Gd II & 3 & 0.25 & -0.15 & -0.10 & -0.10 \\
Tb II & 3 & 0.25 & -0.10 & -0.20 & -0.10 \\
Dy II & 3 & 0.25 & -0.15 & -0.15 & -0.10 \\
Er II & 2 & 0.25 & -0.20 & -0.20 & -0.20 \\
\hline
\end{tabular}

suggestion has arisen that posits that $\mathrm{Ti}$ is an Fe-peak element instead of a classical $\alpha$-element (Sneden et al. 2016). The abundances of stars found in Hor I may lend additional credence to that argument.

Other studies have discovered extremely metal-poor stars having peculiar abundances: Cohen et al. (2008) and Haschke et al. (2012) report discoveries of extremely low metallicity, low- $\alpha$ stars; Caffau et al. (2013) found four extremely metalpoor stars $([\mathrm{Fe} / \mathrm{H}] \sim-3.7)$ with even lower $[\alpha / \mathrm{Fe}]$ ratios than we measure in Hor I. Each of these studies invokes various theoretical SN yield models to explain the observed abundance patterns, which are plausible explanations but in most cases do not perfectly match the observations.

More recently, a metal-poor $([\mathrm{Fe} / \mathrm{H}]=-2.5)$ star having low $\alpha$ abundances $([\alpha / \mathrm{Fe}] \sim-0.4)$, SDSS J0018-0939, was discovered in the SDSS (Aoki et al. 2014). The authors suggest that this star, whose observed abundance patterns are compared to theoretical nucleosynthetic yield models of a pair-instability supernova (PISN; Heger \& Woosley 2002), may represent the first observational evidence of a PISN. Simon et al. (2015b) found two stars in Sculptor with similar chemical abundances but at an average $[\mathrm{Fe} / \mathrm{H}]$ of $\sim-3.9$, which is much more metalpoor than Hor I. Scl 11_1_4296 had depleted abundances of $\mathrm{Mg}, \mathrm{Ca}$, and $\mathrm{Si}$. Scl 07-50 had similarly low $\mathrm{Ca}$ and $\mathrm{Si}$, but an $\mathrm{Mg}$ abundance that is consistent with the Milky Way halo. They concluded that these stars were the second generation of stars formed in the galaxy and that the chemical signatures were reproducible using Population III SN nucleosynthetic models.

Recent models predict that the earliest stars formed that are still observable today should be very (not extremely) metalpoor stars, with $[\mathrm{Fe} / \mathrm{H}]=-2.5$ and low $\alpha$ abundances. Karlsson et al. (2008) constructed theoretical models for the early chemical enrichment of the Milky Way, showing that the lack of metal-free stars in the Galactic halo that are observable today is in fact expected if the first stars to form in the universe were very massive (Bromm et al. 1999). In their model, the Galactic halo is assembled from stars formed during the assembly of "atomic-cooling halos" centered on minihalos each holding a Population III star. These models also show that stars with this chemical signature of $[\mathrm{Fe} / \mathrm{H}]=-2.5$ and low $\alpha$ abundances should be quite rare, about 1 star in 500 in the Galactic halo. This may not be the case in ultrafaint dwarfs. However, this picture is consistent with hierarchical structure formation, as well as, at least qualitatively, with the number of halo stars discovered to date having similarly peculiar abundance patterns. The halo stars described in the above studies are rare and unusual enough, both in observations and in theoretical models when compared to other halo stars, to warrant special attention. It is therefore quite interesting to find three very similar stars colocated in one low-mass galaxy.
We suggest that those peculiar halo stars could have formed in small galaxies like Hor I, in which pollution by a single PISN occurred early in the star formation history of the galaxy. PISNe, due to the high mass of their progenitors, have a characteristically low $\alpha$-element production and a characteristic odd-even effect in their nucleosynthesis pattern. Therefore, the observed abundance patterns of Aoki et al. (2014) and in Hor I may be due to a PISN (we investigate this in more detail in Section 4.3). Those smaller satellites would then have been accreted into the Milky Way halo, leaving small numbers of halo stars with unusual abundance patterns sprinkled throughout the halo, as is observed.

This scenario is consistent with the idea that the ultrafaint dwarfs are small contributors (by mass) to the accretion history of the Milky Way, as predicted by $\Lambda$ CDM theory, and could perhaps be further confirmed if adequate numbers of similarly peculiar stars were found and their kinematic properties are consistent with having originated in the same accreted satellite. This last suggestion may be testable once Gaia proper motions are added to the measured radial velocities, enabling full position and kinematic information.

\subsection{Extended Star Formation in Hor I?}

One plausible scenario that could explain the chemical abundances of Hor I is an early onset of SNe Ia. In our current understanding of chemical evolution (Tinsley 1979), as a starforming gas cloud collapses, the most massive stars form early, quickly evolving to produce SNe II and thereby enriching the surrounding gas cloud with the $\alpha$-elements $\mathrm{O}, \mathrm{Mg}, \mathrm{Si}, \mathrm{S}, \mathrm{Ca}$, and Ti. The next stars that form in this $\alpha$-rich environment would then be $\alpha$-enhanced stars with typical $[\alpha / \mathrm{Fe}]$ values $\geqslant 0.3$. As the stellar population continues to evolve, at some later time $\mathrm{SNe}$ Ia, which have characteristically low yields in $\alpha$ elements and greater yields of the iron-peak elements $(\mathrm{Cr}, \mathrm{Mn}$, $\mathrm{Fe}, \mathrm{Co}, \mathrm{Ni}, \mathrm{Cu}$ ), begin to dominate nucleosynthesis. The $\mathrm{SNe} \mathrm{Ia}$ then enrich the surrounding environment, thereby lowering the relative abundance of $\alpha$-elements and increasing the abundance of iron-peak elements. Stars formed after the transition between Type II-dominated nucleosynthesis and Type Ia-dominated nucleosynthesis would therefore present abundance ratios closer to the solar ratio $(\alpha / \mathrm{Fe}] \sim 0)$. This process produces a characteristic "knee" in the $[\alpha / \mathrm{Fe}]$ ratios across a range of metallicities, where metallicity, or $[\mathrm{Fe} / \mathrm{H}]$, increases with time as the isolated stellar population enriches itself in iron. In principle, the slope and the position of the knee can provide information about the rate and the time, respectively, at which this transition occurred in a given stellar population. McWilliam (1997) provides a comprehensive description of this story, which describes the observed abundances of stars in the Milky Way halo quite well.

Presumably a similar series of events to that described above occurs in all stellar populations. However, the specifics of the 

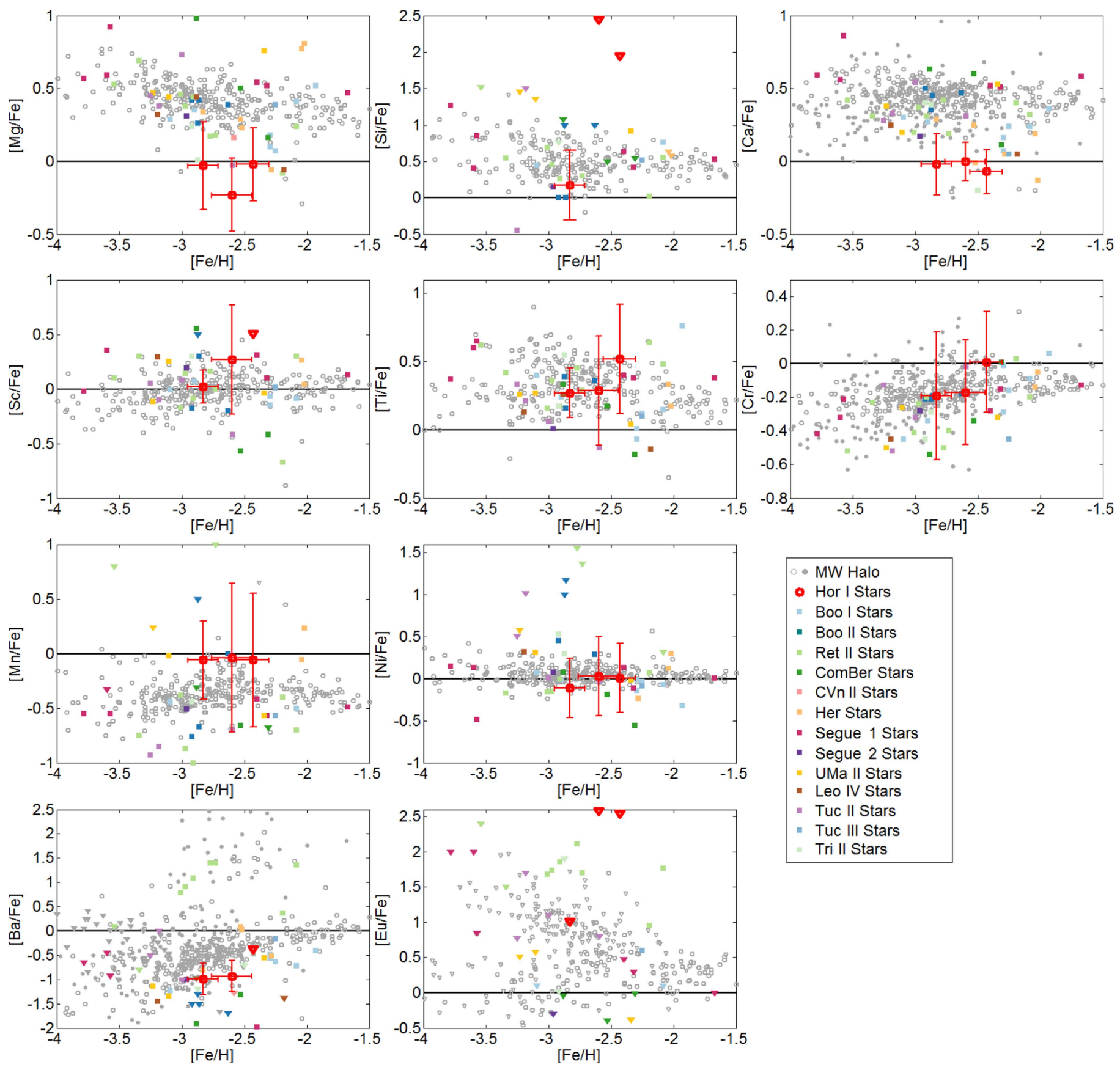

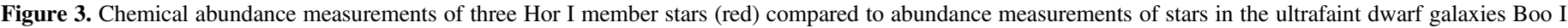

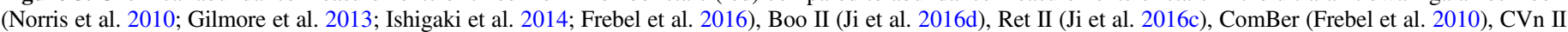

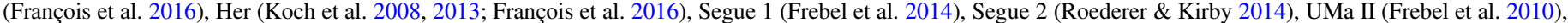

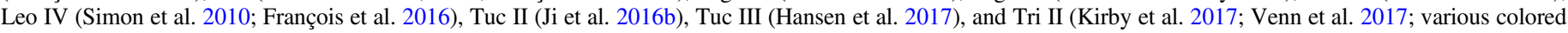

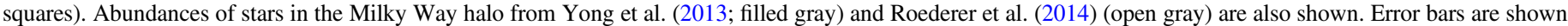
only for the Hor I stars for clarity. Points denoted as $\nabla$ indicate an upper limit. The solar ratio $([\mathrm{X} / \mathrm{Fe}]=0)$ is indicated by the solid black line.

time delay, or, equivalently, metallicity at which this transition between $\alpha$-rich to $\alpha$-poor star formation occurs is determined by the star formation rate and initial mass function of the stellar population. This effect has been observed in dwarf galaxies using both detailed abundance measurements from highresolution spectroscopy (e.g., Venn et al. 2004; Koch et al. 2008; Hendricks et al. 2014) and those from medium-resolution spectroscopy (Kirby et al. 2011). The trend holds for lowermass objects as well: Vargas et al. (2013) studied an ensemble of ultrafaint dwarf galaxies and determined that the transition between Type II-dominated and Type Ia-dominated nucleosynthesis typically occurs in these objects at a "time" when $[\mathrm{Fe} / \mathrm{H}] \sim-2.3$, based on the summary properties of eight ultrafaint dwarfs. According to these results, stars in ultrafaint dwarfs that are more metal-poor than $[\mathrm{Fe} / \mathrm{H}] \sim-2.3$ generally should have formed in the $\alpha$-rich environment produced by $\mathrm{SNe}$ II and thus present supersolar $\alpha$-element abundance. Conversely, stars with $[\mathrm{Fe} / \mathrm{H}]>-2.3$ were produced after $\mathrm{SNe}$ Ia began to pollute the surrounding environment with ironpeak elements and would therefore show $[\alpha / \mathrm{Fe}] \sim 0$. Vargas 
et al. (2013) also determine that star formation in ultrafaint dwarfs occurs after a minimum time delay for the onset of $\mathrm{SNe}$ Ia of at least $100 \mathrm{Myr}$. This picture is consistent with other work that places limits on the star formation histories of ultrafaint dwarf galaxies: deep Hubble Space Telescope imaging and Keck spectroscopy of ultrafaint dwarf galaxies show that their stars were formed early, with roughly $80 \%$ of stars having formed by $12.8 \mathrm{Gyr}$ ago and $100 \%$ of stars formed by $11.6 \mathrm{Gyr}$ ago (Brown et al. 2014). This duration is consistent with an early but extended star formation history that would conform to the standard process of chemical evolution in a stellar population. Furthermore, the picture that has emerged to describe star formation in ultrafaint dwarf galaxies is that star formation began quickly, in some cases in a single burst of star formation (Frebel \& Bromm 2012), and was soon quenched, possibly by reionization (e.g., Brown et al. 2014; Wetzel et al. 2015; Jeon et al. 2017), leaving the stars in the ultrafaint dwarfs as a fossil record of conditions in the early universe.

If we presume a similar chemical evolution timeline for Hor I and use $[\mathrm{Fe} / \mathrm{H}]$ as an age indicator, our measurements imply that the onset of SNe Ia and the subsequent chemical enrichment of the surrounding gas would have had to occur relatively earlier in Hor I than in other ultrafaint dwarfs. The lack of $\alpha$-elements in even the most metal-poor star, DES J025535$540643([\mathrm{Fe} / \mathrm{H}]=-2.8 \pm 0.2$ and $[\mathrm{Ca} / \mathrm{Fe}]=-0.05 \pm 0.15)$, implies that the transition from SN II-dominated nucleosynthesis to SN Ia-dominated nucleosynthesis had to occur at a time when the metallicity of Hor I was $[\mathrm{Fe} / \mathrm{H}]<-2.8$. This would represent a very early transition between nucleosynthesis dominated by SNe II and nucleosynthesis dominated by $\mathrm{SNe}$ Ia compared to other ultrafaint dwarfs.

We do note that it is somewhat presumptuous to draw strong conclusions from a sample of three stars in a galaxy. Furthermore, at least one other ultrafaint dwarf galaxy has shown a spread in $\alpha$-enhancement at the low end of its metallicity range, Ursa Major I (UMa I; Vargas et al. 2013). The 10 stars studied by Vargas et al. (2013) span nearly two orders of magnitude in metallicity with a wide spread in $\alpha$ abundance at the lowest-metallicity end, i.e., UMa I contains at least two metal-poor, $\alpha$-poor stars that could have chemical abundances similar to the Hor I stars. Unfortunately, the moderate-resolution spectroscopy used by Vargas et al. (2013) does not permit detailed abundance analysis of many elements. It should be noted, however, that UMa I may not fit the canonical picture of stellar populations (Jeon et al. 2017).

Limits on the duration of star formation in Hor I could be placed if it were possible to study a larger sample of member stars chemically. According to the standard picture of chemical evolution described above, some of those stars would be older than the three studied here, should have $[\mathrm{Fe} / \mathrm{H}]<-2.8$, and should show $\alpha$-element enhancement consistent with the knee observed in other galaxies. Alternatively, a larger sample of stars could be studied with medium-resolution spectra using techniques such as those used by Kirby et al. (2009).

\subsection{Comparison to Supernova Yield Models}

Alternatively, Hor I may have been host to a rare primordial SN such as a PISN whose nucleosynthetic signature is preserved in the currently observable population of stars. Frebel \& Bromm (2012) suggest that the chemical signatures of low-mass ultrafaint dwarfs can be described by a single, long-
Table 7

Supernova Yield Model Fits to DES J025535-540643

\begin{tabular}{lcc}
\hline \hline Model & $\begin{array}{c}\text { Best-fit } \\
\text { Progenitor Mass }\end{array}$ & $\begin{array}{c}\text { Mean } \\
\text { Sq. Residual }\end{array}$ \\
\hline O Shell $(S=4)$ Piston & $10 M_{\odot}$ & 23.8 \\
Fe Core $\left(Y_{e}\right)$ Piston & $85 M_{\odot}$ & 28.2 \\
PISN & $260 M_{\odot}$ & 64.4 \\
\hline
\end{tabular}

lived generation of stars that formed in the early universe. In related work, Ji et al. (2015) demonstrate that the chemical abundance patterns of these single events can be preserved in the second generation of stars. Though Hor I does not have the characteristically high $\alpha$-element abundance predicted by Frebel \& Bromm (2012) in their "one-shot enrichment" scenario, if Aoki et al. (2014) are correct that their observed abundance patterns, which are similar to ours, are due to a PISN, then we expect that there must have been only a single nucleosynthetic event in Hor I. If there had been several generations of $\mathrm{SNe}$ preceding the currently observed population, the peculiar abundance pattern produced in rare $\mathrm{SNe}$ would be obscured by nucleosynthesis in other, more common SNe II. By this reasoning, for the purposes of this analysis, we assume that the stars in Hor I are chemically primitive objects, and we explore the possibility that the observed abundances could be explained by the predicted yields of a single nucleosynthetic event. Therefore, in our comparison to nucleosynthetic yield models, we limit the number of events to a single Population III SN that enriched the surrounding gas, creating the chemical abundance pattern observed today.

To explore the possibility that the observed abundance pattern of Hor I may arise from a PISN, we have compared the abundances of DES J025535-540643 to various SN yield models for Population III stars. These models can produce low $[\mathrm{Ca} / \mathrm{Fe}]$ and $[\mathrm{Mg} / \mathrm{Fe}]$ abundances, such as those observed in the three stars studied in Hor I. Since we were able to measure more elements in DES J025535-540643, we conduct this analysis only on this star.

We used the STARFIT ${ }^{45}$ tool (C. Chan et al. 2017, in preparation; Heger \& Woosley 2010) to compare our abundance measurements with SN II nucleosynthetic yield models (Heger \& Woosley 2010, and subsequent online updates in 2012) for progenitors spanning a wide range in mass $\left(9.6-100 M_{\odot}\right)$ and PISN nucleosynthetic yield models (Heger \& Woosley 2002) for progenitors spanning a zero-age main-sequence (ZAMS) mass range of 140-260 $M_{\odot}$. The STARFIT code calculates a $\chi^{2}$ statistic using abundance measurements and upper limits (see Heger \& Woosley 2010, Equation (4)) and determines a best-fit SN yield model. We used STARFIT to compare the observed abundance pattern of DES J025535-540643 against three categories of models; we present the parameters of the best-fit models in Table 7. It should be noted that $\mathrm{Sc}$ and $\mathrm{Cr}$ are generally underproduced by yield models. Heger \& Woosley (2010) assume that this is due to additional production sites that are unaccounted for, and therefore discrepancies regarding these elements should be taken lightly. We therefore have STARFIT ignore them when fitting our abundance pattern. Heger \& Woosley (2010) compute yields for nonrotating, metal-free Population III stars using initial big bang compositions from Cyburt et al. (2001).

\footnotetext{
$\overline{{ }^{45} \text { See http://starfit.org for routine and models. }}$
} 

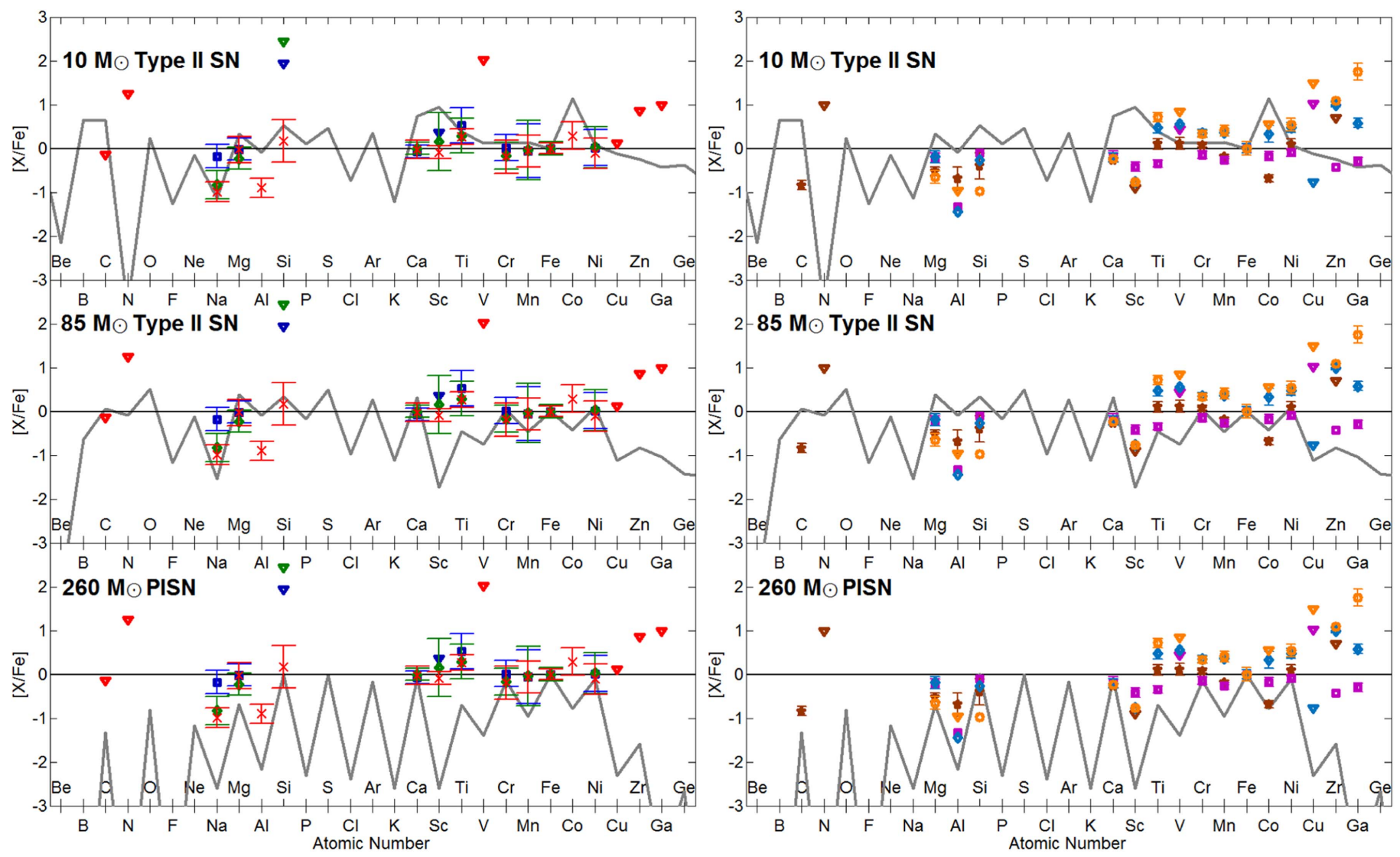

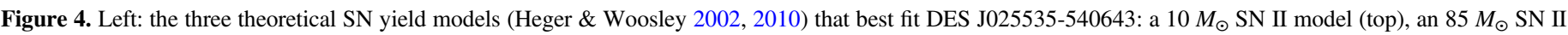

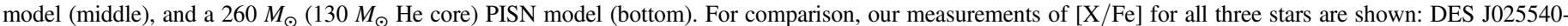

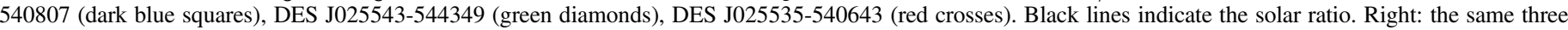

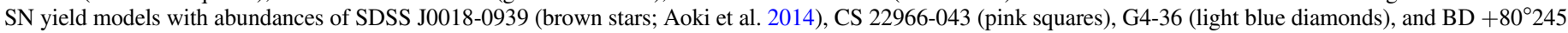
(orange circles; Ivans et al. 2003) shown for comparison. Points denoted as $\nabla$ indicate an upper limit.

Due to the lack of a robust model for how a core-collapse SN explodes, these computations utilize a piston model to simulate the explosion. Heger \& Woosley (2010) compute nucleosynthetic yield models for two locations of the piston (initial mass cut), one model for a piston at the base of the O shell $(S=4$ Piston Model), and one model for a piston at the edge of the $\mathrm{Fe}$ core $\left(Y_{e}\right.$ Piston Model). We compare the abundance of DES J025535-540643 to both models using STARFIT. Using the model for a piston at the base of the $\mathrm{O}$ shell yields a best-fit model of a $10 \quad M_{\odot}$ progenitor SN II (mean squared residual $=23.8$ ). Using the model for a piston at the edge of the Fe core yields a best-fit model of an $85 M_{\odot}$ progenitor SN II (mean squared residual $=28.2$ ).

The explosion mechanism of a PISN is well understood and is simulated to obtain theoretical nucleosynthetic yields by Heger \& Woosley (2002). PISN progenitors enter a regime of electron/positron pair production resulting in a collapse until $\mathrm{O}$ burning and $\mathrm{Si}$ burning produce enough energy to explode. This explosion results in low $\alpha$-element abundances, a low $\mathrm{C}$ abundance, and a strong odd-even effect. Comparison to the model nucleosynthetic yields of PISNe using STARFIT gives a best-fit model of a $260 M_{\odot}\left(130 M_{\odot}\right.$ He core) PISN (mean squared residual $=64.4$ ). It should be noted that this is the highest available PISN model used by STARFIT. It may be that the best-fitting PISN model is beyond the available mass range.

In the left panels of Figure 4, we show the yield models that best fit DES J025535-540643 and the abundance measurements of all three stars observed in Hor I. As can be seen in the figure, each model has difficulties in fitting the observed abundance patterns. The $10 M_{\odot} \mathrm{SN}$ II model produces too much $\mathrm{C}, \mathrm{Ca}$, $\mathrm{Mg}$, and Co compared to our Hor I stars. These four elements produce contradictory requirements. The low upper limit on $\mathrm{C}$ and the abundances of $\mathrm{Ca}$ and $\mathrm{Mg}$ in DES J025535-540643 suggest that a higher-energy explosion than provided by the 10 $M_{\odot} \mathrm{SN}$ II is required, while the low Co abundance requires a lower-energy explosion. The $85 M_{\odot}$ SN II model produces too few iron-peak elements, implying that there is too much fallback and not enough iron-peak elements are synthesized and ejected. This model also does not produce enough Co, indicating that the energy of the explosion is too low. Finally, the $260 M_{\odot}\left(130 M_{\odot}\right.$ He core) PISN model produces a larger odd-even effect and a lower Co abundance than is observed in the stars of Hor I, which show essentially no odd-even effect.

We compare BD $+80^{\circ} 245$, G4-36, CS 22966-043 (Ivans et al. 2003), and SDSS J0018-0939 (Aoki et al. 2014) with the same models that best fit DES J025535-540643 in the right panels of Figure 4. It should be noted that the PISN model that we present is the same model suggested by Aoki et al. (2014) as a possible fit for SDSS J0018-0939. For a common point of comparison for our best-fit models, we also used STARFIT to determine a best-fit PISN model for SDSS J0018-0939. The result was a best-fit model of a $260 M_{\odot}\left(130 M_{\odot}\right.$ He core $)$ PISN (mean squared residual $=159.6)$. Aoki et al. (2014) discussed the discrepancies in this PISN model fit to SDSS J0018-0939, specifically mentioning that the model predicts too much $\mathrm{Si}$ and too large of an odd-even effect for their observed abundance 
pattern. However, the model does fit their measured Co abundance. Our analysis of Hor I shares a similar problem in that the model's predicted odd-even effect is too large for our observed abundance pattern. The model does fit our $\mathrm{Si}$ abundance well, but it underpredicts the amount of Co in DES J025535-540643, mirroring the discrepancies in SDSS J0018-0939.

If it were possible to study a larger sample of member stars chemically, then if a PISN were the underlying cause of the peculiarity in the observed abundance pattern of Hor I, the $\alpha$ element enhancement knee described previously would not be observed. It would require the chemical analysis of many more stars in Hor I to make any strong conclusions.

\subsection{Possible Association with the LMC}

An interesting question posed by the recent discovery of so many candidate ultrafaint dwarf galaxies in the outskirts of the Milky Way and located in the southern hemisphere is whether they originated in the Milky Way or, rather, they originated as satellites of satellites (the Magellanic Clouds). Indeed, both groups announcing the discovery of Hor I (Bechtol et al. 2015; Koposov et al. 2015), as well as the kinematic confirmation work (Koposov et al. 2015), note Hor I's potential association with the LMC due to its location and measured systemic velocity. Several recent theoretical studies have shown that the existence of satellites of satellites is predicted by simulations. Specifically, Deason et al. (2015) use the ELVIS suite of $\mathrm{N}$ body simulations to show that two to four of the nine satellites discovered at the time that were found in close proximity to the LMC are expected to be associated with the LMC, while Sales et al. (2017) use the Aquarius Project suite of zoomed-in cosmological simulations to show that 2-3 of all 46 dwarfs located within $300 \mathrm{kpc}$ of the Milky Way should be associated with the LMC. Both of these works specifically state that Hor I has a high probability of being associated with the LMC according to their simulations. Jethwa et al. (2016) use a complementary approach to these results and construct a dynamical model to determine which, if any, of the DESdiscovered satellites could have Magellanic origins assuming that the Milky Way-LMC system follows the distribution of subhalos predicted by $\Lambda$ CDM. Their model uses the satellites' observed positions and kinematic parameters to show that 7 of the 14 candidate DES satellites in the range $-7<M_{V}<-1$ discovered by Bechtol et al. (2015), Koposov et al. (2015), and Drlica-Wagner et al. (2015) are likely to be satellites of the LMC rather than of the Milky Way. Their simulations produce predicted systemic velocities for the DES satellites, which must be confirmed by spectroscopic follow-up observations (only 4 of the 14 had measured velocities at the time of writing: Hor I, Ret II, Gru I, and Tuc II). To date, of the satellites considered by Jethwa et al. (2016), Hor I's measured systemic velocity would be by far the closest to the velocity predicted if Hor I were associated with the LMC.

If Hor I is indeed a satellite of the LMC, the chemical abundance pattern of Hor I could provide further interesting information about the relationship of the satellite to its host. The LMC has an overall lower $\alpha$-enhancement than the Milky Way (e.g., Pompéia et al. 2008; Lapenna et al. 2012; Van der Swaelman et al. 2013). Van der Swaelman et al. (2013) suggest that the lack of $\alpha$-elements implies a significantly different star formation history for the LMC than that of the Milky Way halo. Hence, the lower $\alpha$-abundance of the Hor I stars may simply be due to its Magellanic origin and the fact that early star formation in the LMC proceeded quite differently than in the halo of the Milky Way. The detailed abundance analysis of additional stars in Hor I, as well as of other candidate satellites of the LMC, would lend credence to this hypothesis. However, with only the three stars observed in this study, the chemical abundance pattern of Hor I does not exclude the possibility of an association with the LMC, nor does it strongly suggest it. The strongest evidence that Hor I is a satellite of the LMC is the measured radial velocity of its member stars.

\section{Conclusions}

We have measured the chemical abundances of three confirmed member stars in Hor I and have shown that it is yet another example of an ultrafaint dwarf galaxy having a peculiar abundance pattern. Hor I's average metallicity of $[\mathrm{Fe} / \mathrm{H}] \sim-2.6$ is not particularly exceptional; however, the observed $\alpha$ abundances are much lower than expected when compared to other metal-deficient stars. In addition, the abundances of other elements, in particular the iron-peak elements, are close to the solar ratio, which is unusually high when compared to most Milky Way halo stars. We put forward the possibility that Hor I could have the earliest known transition between nucleosynthesis dominated by SNe II and nucleosynthesis dominated by SNe Ia. Alternatively, Hor I's chemistry could be explained by a PISN, or it could be a satellite of the LMC. In either case, our small sample of three stars is not enough to confirm these suggestions, and additional member stars must be studied.

Four DES-discovered ultrafaint dwarfs have been chemically studied in detail to date: Ret II (Ji et al. 2016a; Roederer et al. 2016), Tuc II (Ji et al. 2016b), Tuc III (Hansen et al. 2017), and now Hor I. In each case (with the possible exception of Tuc II), the brightest confirmed member stars show an unexpected and peculiar abundance pattern. Although a plausible explanation for the observed abundances in each system can be invoked, the variety of explanations is large, suggesting that star formation processes in the early universe may be highly stochastic. These results suggest that study of additional ultrafaint dwarfs, as well as additional stars in these four previously studied ultrafaint dwarfs, may shed more light on how the first stars and galaxies were formed. However, probing the detailed chemical abundance patterns in many more confirmed member stars in Hor I will likely not be possible until the next generation of telescopes comes online in the next decade.

D.Q.N. wishes to thank the referee, Chris Sneden, for his insightful comments. He also thanks Katelyn Stringer for editorial comments. E.B. acknowledges financial support from the European Research Council (ERC-StG-335936). C.M.P. was supported by NSF grant AST-1560223, "REU Site: Astronomical Research and Instrumentation at Texas A\&M University."

Funding for the DES Projects has been provided by the U.S. Department of Energy, the U.S. National Science Foundation, the Ministry of Science and Education of Spain, the Science and Technology Facilities Council of the United Kingdom, the Higher Education Funding Council for England, the National Center for Supercomputing Applications at the University of Illinois at Urbana-Champaign, the Kavli Institute of Cosmological Physics at the University of Chicago, the Center for 
Cosmology and Astro-Particle Physics at the Ohio State University, the Mitchell Institute for Fundamental Physics and Astronomy at Texas A\&M University, Financiadora de Estudos e Projetos, Fundação Carlos Chagas Filho de Amparo à Pesquisa do Estado do Rio de Janeiro, Conselho Nacional de Desenvolvimento Científico e Tecnológico and the Ministério da Ciência, Tecnologia e Inovação, the Deutsche Forschungsgemeinschaft, and the Collaborating Institutions in the Dark Energy Survey.

The Collaborating Institutions are Argonne National Laboratory, the University of California at Santa Cruz, the University of Cambridge, Centro de Investigaciones Energéticas, Medioambientales y Tecnológicas-Madrid, the University of Chicago, University College London, the DES-Brazil Consortium, the University of Edinburgh, the Eidgenössische Technische Hochschule (ETH) Zürich, Fermi National Accelerator Laboratory, the University of Illinois at UrbanaChampaign, the Institut de Ciències de l'Espai (IEEC/CSIC), the Institut de Física d'Altes Energies, Lawrence Berkeley National Laboratory, the Ludwig-Maximilians Universität München and the associated Excellence Cluster Universe, the University of Michigan, the National Optical Astronomy Observatory, the University of Nottingham, The Ohio State University, the University of Pennsylvania, the University of Portsmouth, SLAC National Accelerator Laboratory, Stanford University, the University of Sussex, Texas A\&M University, and the OzDES Membership Consortium.

Based in part on observations at Cerro Tololo InterAmerican Observatory, National Optical Astronomy Observatory, which is operated by the Association of Universities for Research in Astronomy (AURA) under a cooperative agreement with the National Science Foundation.

The DES data management system is supported by the National Science Foundation under grant nos. AST-1138766 and AST-1536171. The DES participants from Spanish institutions are partially supported by MINECO under grants AYA2015-71825, ESP2015-88861, FPA2015-68048, SEV2012-0234, SEV-2016-0597, and MDM-2015-0509, some of which include ERDF funds from the European Union. IFAE is partially funded by the CERCA program of the Generalitat de Catalunya. Research leading to these results has received funding from the European Research Council under the European Union's Seventh Framework Program (FP7/20072013), including ERC grant agreements 240672, 291329, and 306478. We acknowledge support from the Australian Research Council Centre of Excellence for All-sky Astrophysics (CAASTRO), through project no. CE110001020.

This manuscript has been authored by Fermi Research Alliance LLC under contract no. DE-AC02-07CH11359 with the U.S. Department of Energy, Office of Science, Office of High Energy Physics. The U.S. Government retains and the publisher, by accepting the article for publication, acknowledges that the U.S. Government retains a nonexclusive, paidup, irrevocable, worldwide license to publish or reproduce the published form of this manuscript, or allow others to do so, for U.S. Government purposes.

IRAF is distributed by the National Optical Astronomy Observatory, which is operated by the Association of Universities for Research in Astronomy (AURA) under a cooperative agreement with the National Science Foundation.

This paper has gone through internal review by the DES Collaboration.

\section{ORCID iDs}

J. L. Marshall (iD https://orcid.org/0000-0003-0710-9474

T. T. Hansen (i) https://orcid.org/0000-0001-6154-8983

A. Drlica-Wagner (iD https://orcid.org/0000-0001-8251-933X

L. E. Strigari (i) https://orcid.org/0000-0001-5672-6079

A. R. Walker (1) https://orcid.org/0000-0002-7123-8943

J. Annis (i) https://orcid.org/0000-0002-0609-3987

A. Carnero Rosell (iD https://orcid.org/0000-0003-3044-5150

D. W. Gerdes (iD https://orcid.org/0000-0001-6942-2736

D. Gruen (iD https://orcid.org/0000-0003-3270-7644

R. A. Gruendl (iD https://orcid.org/0000-0002-4588-6517

R. Miquel (iD https://orcid.org/0000-0002-6610-4836

A. Roodman (iD https://orcid.org/0000-0001-5326-3486

M. Soares-Santos (i) https://orcid.org/0000-0001-6082-8529

G. Tarle (i) https://orcid.org/0000-0003-1704-0781

R. H. Wechsler (iD https://orcid.org/0000-0003-2229-011X

\section{References}

Aoki, W., Tominaga, N., Beers, T. C., Honda, S., \& Lee, Y. S. 2014, Sci, 345,912

Asplund, M., Grevesse, N., Sauval, A. J., \& Scott, P. 2009, ARA\&A, 47, 481

Bechtol, K., Drlica-Wagner, A., Balbinot, E., et al. 2015, ApJ, 807, 50

Beers, T. C., \& Christlieb, N. 2005, ARA\&A, 43, 531

Belokurov, V. 2013, NewAR, 57, 100

Belokurov, V., Zucker, D. B., Evans, N. W., et al. 2006, ApJL, 642, L137

Bernstein, R., Shectman, S. A., Gunnels, S. M., Mochnacki, S., \& Athey, A. E. 2003, Proc. SPIE, 4841, 1694

Biémont, É, Blagoev, K., Engström, L., et al. 2011, MNRAS, 414, 3350

Biemont, E., Grevesse, N., Hannaford, P., \& Lowe, R. M. 1981, ApJ, 248, 867

Boylan-Kolchin, M., Bullock, J. S., \& Kaplinghat, M. 2011, MNRAS, 415, L40

Bromm, V., Coppi, P. S., \& Larson, R. B. 1999, ApJL, 527, L5

Brown, T. M., Tumlinson, J., Geha, M., et al. 2014, ApJ, 796, 91

Bullock, J. S., \& Johnston, K. V. 2005, ApJ, 635, 931

Caffau, E., Bonifacio, P., François, P., et al. 2013, A\&A, 560, A15

Carney, B. W., Wright, J. S., Sneden, C., et al. 1997, AJ, 114, 363

Castelli, F., \& Kurucz, R. L. 2004, arXiv:astro-ph/0405087

Cohen, J. G., Christlieb, N., McWilliam, A., et al. 2008, ApJ, 672, 320

Cyburt, R. H., Fields, B. D., \& Olive, K. A. 2001, NewA, 6, 215

Deason, A. J., Wetzel, A. R., Garrison-Kimmel, S., et al. 2015, MNRAS, 453,3568

Dekker, H., D’Odorico, S., Kaufer, A., Delabre, B., \& Kotzlowski, H. 2000, Proc. SPIE, 4008, 534

Den Hartog, E. A., Lawler, J. E., Sneden, C., \& Cowan, J. J. 2003, ApJS, 148,543

Den Hartog, E. A., Lawler, J. E., Sneden, C., \& Cowan, J. J. 2006, ApJS, 167,292

Den Hartog, E. A., Lawler, J. E., Sobeck, J. S., Sneden, C., \& Cowan, J. J. 2011, ApJS, 194, 35

Dotter, A., Chaboyer, B., Jevremović, D., et al. 2008, ApJS, 178, 89

Drlica-Wagner, A., Bechtol, K., Rykoff, E. S., et al. 2015, ApJ, 813, 109

François, P., Monaco, L., Bonifacio, P., et al. 2016, A\&A, 588, A7

Frebel, A. 2010, AN, 331, 474

Frebel, A., \& Bromm, V. 2012, ApJ, 759, 115

Frebel, A., Casey, A. R., Jacobson, H. R., \& Yu, Q. 2013, ApJ, 769, 57

Frebel, A., \& Norris, J. 2015, ARA\&A, 53, 631

Frebel, A., Norris, J. E., Gilmore, G., \& Wyse, R. F. G. 2016, ApJ, 826, 110

Frebel, A., Simon, J. D., Geha, M., \& Willman, B. 2010, ApJ, 708, 560

Frebel, A., Simon, J. D., \& Kirby, E. N. 2014, ApJ, 786, 74

Gallagher, A. J. 2012, PhD thesis, Univ. Hertfordshire

Gallagher, A. J., Ryan, S. G., García Pérez, A. E., \& Aoki, W. 2010, A\&A, 523, A24

Gilmore, G., Norris, J. E., Monaco, L., et al. 2013, ApJ, 763, 61

Hansen, T. T., Simon, J. D., Marshall, J. L., et al. 2017, ApJ, 838, 44

Haschke, R., Grebel, E. K., Frebel, A., et al. 2012, AJ, 144, 88

Heger, A., \& Woosley, S. E. 2002, ApJ, 567, 532

Heger, A., \& Woosley, S. E. 2010, ApJ, 724, 341

Hendricks, B., Koch, A., \& Lanfranchi, G. A. 2014, ApJ, 785, 102

Ishigaki, M. N., Aoki, W., Arimoto, N., \& Okamoto, S. 2014, A\&A, 562, A146

Ivans, I. I., Sneden, C., James, C. R., et al. 2003, AJ, 592, 906

Jeon, M., Besla, G., \& Bromm, V. 2017, ApJ, 848, 85 
Jethwa, P., Erkal., D., \& Belokurov, V. 2016, MNRAS, 461, 2212 Ji, A. P., Frebel, A., \& Bromm, V. 2015, MNRAS, 454, 659 Ji, A. P., Frebel, A., Chiti, A., \& Simon, J. D. 2016a, Natur, 531, 610 Ji, A. P., Frebel, A., Ezzeddine, R., \& Casey, A. R. 2016b, ApJL, 832, L3 Ji, A. P., Frebel, A., Simon, J. D., \& Chiti, A. 2016c, ApJ, 830, 93 Ji, A. P., Frebel, A., Simon, J. D., \& Geha, M. 2016d, ApJ, 817, 41 Johnston, K. V., Bullock, J. S., Sharma, S., et al. 2008, ApJ, 689, 936 Karlsson, T., Johnson, J. L., \& Bromm, V. 2008, ApJ, 679, 6

Kelson, D. D. 2003, PASP, 115, 688

Kim, D., \& Jerjen, H. 2015, ApJL, 808, L39

Kim, D., Jerjen, H., Milone, A. P., et al. 2015, ApJ, 803, 63

Kirby, E. N., Cohen, J. G., Simon, J. D., et al. 2017, ApJ, 838, 83

Kirby, E. N., Cohen, J. G., Smith, G. H., et al. 2011, ApJ, 727, 79

Kirby, E. N., Guhathakurta, P., Bolte, M., et al. 2009, ApJ, 705, 328

Klypin, A., Kravtsov, A. V., Valenzuela, O., et al. 1999, ApJ, 522, 82

Koch, A., Feltzing, S., Adén, D., \& Matteucci, F. 2013, A\&A, 554, A5

Koch, A., Grebel, E. K., Gilmore, G. F., et al. 2008, AJ, 135, 1580

Koch, A., McWilliam, A., Grebel, E. K., Zucker, D. B., \& Belokurov, V. 2008, ApJL, 688, L13

Koposov, S. E., Belokurov, V., Torrealba, G., \& Evans, N. W. 2015, ApJ, 805,130

Koposov, S. E., Casey, A. R., Belokurov, V., et al. 2015, ApJ, 811, 62

Kramida, A., Ralchenko, Yu., Reader, J. \& NIST ASD Team 2016, NIST Atomic Spectra Database (version 5.4), http://physics.nist.gov/asd

Kurucz, R. L. 2011, CaJPh, 89, 417

Kurucz, R. L., \& Bell, B. (ed.) 1995, Atomic Line Data (Cambridge, MA: Smithsonian Astrophysical Observatory)

Lapenna, E., Mucciarelli, A., Origlia, L., \& Ferraro, F. R. 2012, ApJ, 761, 33

Lawler, J. E., Bonvallet, G., \& Sneden, C. 2001a, ApJ, 556, 452

Lawler, J. E., \& Dakin, J. T. 1989, JOSAB, 6, 1457

Lawler, J. E., Den Hartog, E. A., Sneden, C., \& Cowan, J. J. 2006, ApJS, 162,227

Lawler, J. E., Guzman, A., Wood, M. P., Sneden, C., \& Cowan, J. J. 2013, ApJS, 205, 11

Lawler, J. E., Sneden, C., \& Cowan, J. J. 2015, ApJS, 220, 13

Lawler, J. E., Sneden, C., Cowan, J. J., et al. 2008, ApJS, 178, 71

Lawler, J. E., Sneden, C., Cowan, J. J., Ivans, I. I., \& Den Hartog, E. A. 2009, ApJS, 182, 51

Lawler, J. E., Wickliffe, M. E., Cowley, C. R., \& Sneden, C. 2001b, ApJS, 137,341

Lawler, J. E., Wickliffe, M. E., den Hartog, E. A., \& Sneden, C. 2001c, ApJ, 563,1075

Lawler, J. E., Wood, M. P., Den Hartog, E. A., et al. 2014, ApJS, 215, 20

Lawler, J. E., Wyart, J.-F., \& Blaise, J. 2001d, ApJS, 137, 351

Li, R., Chatelain, R., Holt, R. A., et al. 2007, PhyS, 76, 577

Li, T. S., Simon, J. D., Drlica-Wagner, A., et al. 2017, ApJ, 838, 8

Lind, K., Asplund, M., Barklem, P. S., \& Belyaev, A. K. 2011, A\&A, 528, A103

Luque, E., Pieres, A., \& Santiago, B. 2016a, MNRAS, 468, 97

Luque, E., Queiroz, A., \& Santiago, B. 2016b, MNRAS, 458, 603

Massarotti, A., Latham, D. W., Stefanik, R. P., \& Fogel, J. 2008, AJ, 135, 209

Masseron, T., Plez, B., Van Eck, S., et al. 2014, A\&A, 571, A47
McConnachie, A. W. 2012, AJ, 144, 4

McWilliam, A. 1997, ARA\&A, 35, 503

McWilliam, A., \& Rich, R. M. 1994, ApJS, 91, 749

Modigliani, A., Mulas, G., Porceddu, I., et al. 2004, Msngr, 118, 8

Moore, B. 1994, Natur, 370, 629

Norris, J. E., Wyse, R. F. G., Gilmore, G., et al. 2010, ApJ, 723, 1632

Palmeri, P., Fischer, C. F., Wyart, J.-F., \& Godefroid, M. R. 2005, MNRAS, 363, 452

Pasquini, L., Avila, G., Allaert, E., et al. 2000, Proc. SPIE, 4008, 129

Pompéia, L., Hill, V., Spite, M., et al. 2008, A\&A, 480, 379

Robertson, B., Bullock, J. S., Font, A. S., Johnston, K. V., \& Hernquist, L. 2005, ApJ, 632, 872

Roederer, I. U., \& Kirby, E. N. 2014, MNRAS, 440, 2665

Roederer, I. U., Mateo, M., Bailey, J. I., III, et al. 2016, AJ, 151, 82

Roederer, I. U., Preston, G. W., Thompson, I. B., et al. 2014, AJ, 147, 136

Ruffoni, M. P., Den Hartog, E. A., Lawler, J. E., et al. 2014, MNRAS, 441, 3127

Sales, L. V., Navarro, J. F., Kallivayalil, N., et al. 2017, MNRAS, 465, 1879

Searle, L., \& Zinn, R. 1978, ApJ, 225, 357

Simon, J. D., Drlica-Wagner, A., Li, T. S., et al. 2015a, ApJ, 808, 95

Simon, J. D., Frebel, A., McWilliam, A., Kirby, E. N., \& Thompson, I. B. 2010, ApJ, 716, 446

Simon, J. D., \& Geha, M. 2007, ApJ, 670, 313

Simon, J. D., Jacobson, H. R., Frebel, A., et al. 2015b, ApJ, 802, 93JLM

Simon, J. D., Li, T. S., Drlica-Wagner, A., et al. 2017, ApJ, 838, 11

Sneden, C. 1973, ApJ, 184, 839

Sneden, C., Cowan, J. J., \& Gallino, R. 2008, ARA\&A, 46, 241

Sneden, C., Cowan, J. J., Kobayashi, C., et al. 2016, ApJ, 817, 53

Sneden, C., Lawler, J. E., Cowan, J. J., Ivans, I. I., \& Den Hartog, E. A. 2009, ApJS, 182, 80

Sneden, C., Lucatello, S., Ram, R. S., Brooke, J. S. A., \& Bernath, P. 2014, ApJS, 214, 26

Sneden, C., Uomoto, A., Cottrell, P., \& Fitzpatrick, M. 2012, SPECTRE: Manipulation of Single-order Spectra, Astrophysics Source Code Library, ascl: 1202.010

Sobeck, J. S., Kraft, R. P., Sneden, C., et al. 2011, AJ, 141, 175

Sobeck, J. S., Lawler, J. E., \& Sneden, C. 2007, ApJ, 667, 1267

The Dark Energy Survey Collaboration 2005, arXiv:astro-ph/0510346

Tinsley, B. 1979, ApJ, 229, 1046

Van der Swaelman, M., Hill, V., Primas, F., \& Cole, A. A. 2013, A\&A, 560, A44

Vargas, L. C., Geha, M., Kirby, E. N., \& Simon, J. D. 2013, ApJ, 767, 134

Venn, K. A., Irwin, M., Shetrone, M. D., et al. 2004, AJ, 128, 1177JLM

Venn, K. A., Starkenburg, E., Malo, L., Martin, N., \& Laevens, B. P. M. 2017, MNRAS, 466, 3741

Walker, M. G., Mateo, M., Olszewski, E. W., et al. 2016, ApJ, 819, 53

Wetzel, A. R., Deason, A. J., \& Garrison-Kimmel, S. 2015, ApJ, 807, 49

Wickliffe, M. E., Lawler, J. E., \& Nave, G. 2000, JQSRT, 66, 363

Wickliffe, M. E., Salih, S., \& Lawler, J. E. 1994, JQSRT, 51, 545

Willman, B., \& Strader, J. 2012, AJ, 144, 76

Wood, M. P., Lawler, J. E., Sneden, C., \& Cowan, J. J. 2014, ApJS, 211, 20

Yong, D., Norris, J. E., Bessell, M. S., et al. 2013, ApJ, 762, 26 